\title{
Spectroscopy of very low mass stars and brown dwarfs in the Lambda Orionis star forming region
}

\section{Enlarging the census down to the planetary mass domain in Collinder $69 \star, \star \star$}

\author{
A. Bayo ${ }^{1,3}$, D. Barrado ${ }^{2,3}$, J. Stauffer ${ }^{4}$, M. Morales-Calderón ${ }^{3,4}$, C. Melo ${ }^{1}$, N. Huélamo ${ }^{3}$, \\ H. Bouy ${ }^{3}$, B. Stelzer ${ }^{5}$, M. Tamura ${ }^{6}$, and R. Jayawardhana ${ }^{7}$ \\ ${ }^{1}$ European Southern Observatory, Av. Alonso de Córdova 3107, Santiago 19 Santiago, Chile \\ e-mail: abayo@eso.org \\ 2 Calar Alto Observatory, Centro Astronómico Hispano Alemán, C/Jesús Durbán Remón, 2-2 04004 Almería, Spain \\ 3 Depto. Astrofísica, Centro de Astrobiología (INTA-CSIC), PO Box 78, 28691 Villanueva de la Cañada, Spain \\ ${ }^{4}$ Spitzer Science Center, California Institute of Technology, Pasadena, CA 91125 \\ 5 INAF - Osservatorio Astronomico di Palermo, Piazza del Parlamento 1, 90134 Palermo, Italy \\ ${ }^{6}$ National Astronomical Observatory, 2-21-1 Osawa, Mitaka, Tokyo 181-8588, Japan \\ 7 Department of Astronomy and Astrophysics, University of Toronto, Toronto, ON M5S 3H8, Canada
}

Received 1 February 2011 / Accepted 21 september 2011

\section{ABSTRACT}

\begin{abstract}
Context. Whilst there is a generally accepted evolutionary scheme for the formation of low-mass stars, the analogous processes when moving down in mass to the brown dwarf regime are not yet well understood.

Aims. In this first paper, we try to compile the most complete and unbiased spectroscopically confirmed census of the population of Collinder 69, the central cluster of the Lambda Orionis star forming region, as a first step in addressing the question of how brown dwarfs and planetary mass objects form.

Methods. We study age dependent features in optical and near-infrared spectra of candidate members to the cluster (such as alkali lines and accretion-associated indicators). In addition, we complement that study with the analysis of other youth indicators, such as X-ray emission or mid-infrared excess.

Results. We confirm the membership to Collinder 69 of $\sim 90$ photometric candidate members. As a byproduct, we determine a temperature scale for young $M$, very low-mass stars, and brown dwarfs. We assemble one of the most complete initial mass functions from 0.016 to $20 M_{\odot}$. Finally, we study the implications of the spatial distribution of the confirmed members for the proposed mechanisms of brown dwarf formation.
\end{abstract}

Key words. brown dwarfs - stars: formation - stars: luminosity function, mass function - infrared: stars - stars: low-mass open clusters and associations: individual: Collinder 69

\section{Introduction}

In the current paradigm, stars are born within molecular clouds, which are accumulations of gas and dust. These clouds are initially supported against gravitational collapse by a combination of thermal, magnetic, and turbulent pressure (Shu et al. 1987; Mouschovias 1991). Nevertheless, molecular clouds can fragment into smaller and denser cores, where the presence of gravitational instabilities causes the collapse of the cloud material (Shu et al. 1987, and references therein).

Brown dwarfs are very low mass objects characterized by the lack of stable hydrogen burning in their interior and masses typically below $0.072 M_{\odot}$. In 1995 , their discovery led to a debate about the formation mechanism behind this type of objects that is ongoing to this day. Since the typical thermal Jeans mass in molecular cloud cores is around $1 M_{\odot}$, a thermally supported

\footnotetext{
* Appendix A is available in electronic form at http: //www . aanda.org

$\star \star$ Table 6 is also available in electronic form at the CDS via anonymous ftp to cdsarc.u-strasbg. fr $(130.79 .128 .5)$ or via http://cdsarc.u-strasbg.fr/viz-bin/qcat?J/A+A/536/A63
}

cloud does not fragment in cores of substellar masses and the formation of brown dwarfs cannot be directly explained as a scaleddown version of low-mass star formation.

While Padoan \& Nordlund (2002) argued that brown dwarfs form via "turbulent fragmentation" (the density enhancements produced by the turbulence that lead to a decrease in the Jeans mass), Reipurth \& Clarke (2001) suggested that they may be stellar embryos ejected from newborn multiple systems before they accreted enough mass to start hydrogen burning (in this model, the truncation of the disks is explained by dynamical interactions). In addition, Whitworth \& Zinnecker (2004) proposed photo-evaporation of massive pre-stellar cores as the formation mechanism of brown dwarfs.

Even though a significant number of brown dwarfs with ages around a few Myr have been reported to harbor active disks (Luhman et al. 1997; Fernández \& Comerón 2001; Natta et al. 2004; Barrado y Navascués \& Martín 2003; Barrado y Navascués et al. 2004a; Mohanty et al. 2005) favoring the "in-situ" formation scenario, more homogeneous and systematic studies of disk accretion, rotation, and activity (and the 
relationship between classic indicators of activity such as $\mathrm{H} \alpha$ and these phenomena) in young brown dwarfs could help us to confirm the "universality" of this mechanism or the dependence on other (environmental) factors.

These kinds of studies can only be carried when based on a robust census (for masses well below the hydrogen burning limit) of star forming regions of different ages and environments. This work presents such a census for a very interesting star forming region, and, by studying the spatial distribution of its members, addresses the question on the mechanism behind the formation of brown dwarfs in this particular environment.

The Lambda Orionis star forming region (LOSFR) is associated with the O8III star $\lambda$ Orionis (located at $\sim 400 \mathrm{pc}$, Murdin $\&$ Penston 1977), the head of the Orion giant. It comprises both recently formed stars up to $\sim 24 M_{\odot}$ and dark clouds actively forming stars. Although its properties (morphology, distance, reddening, size) ensure that this region of star formation is an ideal laboratory for testing star formation theories, until recently, it had not been very well-studied.

Duerr et al. (1982) carried out an $\mathrm{H} \alpha$ emission survey identifying three stellar clusters centered around the dark clouds Barnard 30 and Barnard 35. Those clusters were later confirmed and analyzed from a statistical point of view by Gomez \& Lada (1998). In particular, Collinder 69, the central one, is a welldefined, compact open cluster affected by rather low extinction $A_{v} \sim 0.36 \mathrm{mag}$ (Duerr et al. 1982). It is quite rich, containing one O binary star ( $\lambda$ Ori itself), about a dozen B stars (Duerr et al. 1982), and a well-populated sequence of low-mass stars and brown dwarfs (Barrado y Navascués et al. 2004b, 2007).

A number of photometric studies have been published focused on Collinder 69: Dolan \& Mathieu (1999, 2001, 2002) obtained optical photometry and presented a selection of candidates with estimated masses as low as $\sim 0.3 M_{\odot}$ (assuming an age of $5 \mathrm{Myr}$ ). Morales-Calderón (2008) presented a very complete compilation of photometry from the optical to the mid-IR obtained with different ground-based and space observatories (including previous candidates by Barrado y Navascués et al. 2004b, 2007). They provided a list of candidates with estimated masses well below the hydrogen burning boundary if membership to the cluster is confirmed. This sample (together with the X-ray candidates, see next section) represents the starting point of this work. Our aim here is the spectroscopic characterization and confirmation of membership. When we combine our membership results with those of other spectroscopic works (Dolan \& Mathieu 1999, 2001; Barrado y Navascués et al. 2004b; Sacco et al. 2008; Maxted et al. 2008), we find a pollution rate of only $\sim 9 \%$; this suggests that the methods followed by Morales-Calderón (2008) to obtain candidate members are very reliable.

This paper is organized as follows. In Sect. 2, we describe very briefly the photometric and X-ray data from which the candidate selection was obtained by Morales-Calderón (2008) and Barrado et al. (2011). In Sect. 3, we describe the spectroscopic observations of the candidates. In Sect. 4, we present a temperature scale derived for $M$ young very-low-mass stars and brown dwarfs (from our spectroscopically confirmed members of Collinder 69). In Sect. 5, we describe the procedure followed to build our final census of members. Finally, in Sects. 6 and 7 we present the study of the spatial distribution of the confirmed members (and its implications for the theories of the formation of brown dwarfs) and one of the most complete initial mass functions constructed so far, respectively. Our conclusions are summarized in Sect. 8.

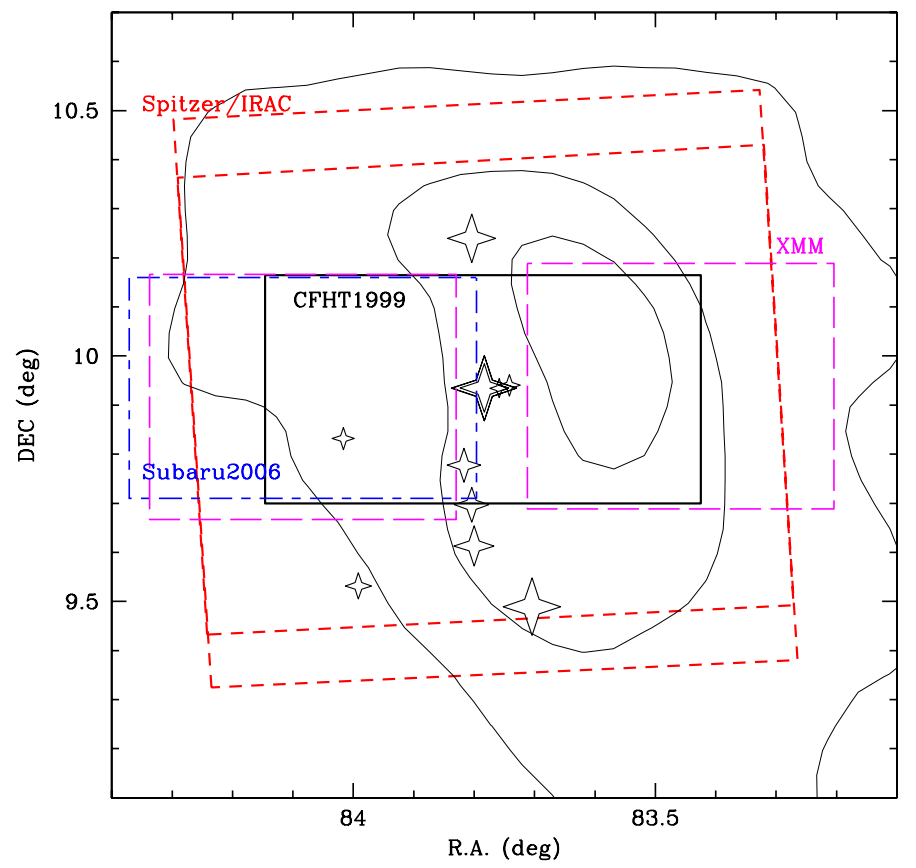

Fig. 1. Sketch of the areas covered by the main optical, infrared, and $\mathrm{X}$-ray surveys in the region of Collinder 69. $\lambda$ Ori itself is highlighted with a large star and the B population with smaller ones. Omega 2000 and Ingrid data together fill the area covered by the CFHT survey (we do not include the mosaics for the sake of clarity in the figure). The large red dashed boxes show the IRAC FoV - the edges are the regions with coverage only in 3.6/5.8 or 4.5/8.0 - and the area surveyed by MIPS is slightly larger.

On a forthcoming paper (Bayo et al., in prep., from now on Paper II), we present the properties of individual members of Collinder 69 (such as accretion rates, rotational velocities, etc.), as well as more general cluster-related ones (such as disk ratios and disk spatial distribution).

\section{Photometric candidates in Collinder 69}

As mentioned before, our study here is based on the followup of the photometric candidates proposed in Morales-Calderón (2008) and a sample of the X-ray emitters reported in Barrado et al. (2011). Here we provide a short description of the data used in these two works and in Fig. 1 we show a diagram with the coverage of the different surveys over the contours of the $100 \mu \mathrm{m}$ IRAS image of the cluster:

\section{- Optical:}

1. The CFHT1999 Survey (Cousins $R$ and $I$ bands): described in Barrado y Navascués et al. (2004b), for cluster members, the faint limit is set at $R_{\text {Complete }} \sim 22.75 \mathrm{mag}$ at $(R-I)=2.5$, corresponding then to $I_{\text {Complete,cluster }} \sim$ $20.2 \mathrm{mag}$. For a DUSTY 5 Myr isochrone (Chabrier et al. 2000), and the distance and standard extinction for the cluster, this limit corresponds to $20 M_{\text {Jupiter }}$.

2. The Subaru2006 Survey ( $i^{\prime}$ and $z^{\prime}$ bands): described in Morales-Calderón (2008), for a DUSTY 5 Myr isochrone (Chabrier et al. 2000), and the distance and standard extinction for the cluster, an I magnitude of 25.5 mag corresponds to $\sim 7 M_{\text {Jupiter }}$; for a COND $5 \mathrm{Myr}$ isochrone, which is more appropriate for this range of temperatures, the completeness limit is located at $3 M_{\text {Jupiter }}$. 
Table 1. A summary of the Collinder 69 optical spectroscopic campaigns.

\begin{tabular}{|c|c|c|c|c|c|}
\hline Date & Observatory/Telescope/Instrument & $\begin{array}{c}\text { Resolution } \\
\Delta \lambda / \lambda\end{array}$ & $\begin{array}{l}\text { Wavelength } \\
\text { coverage }\end{array}$ & $\begin{array}{c}\text { Number of sources } \\
\text { observed }\end{array}$ & $\begin{array}{c}\text { Original photometric } \\
\text { survey }\end{array}$ \\
\hline Nov. 2-5, 2002 & Mauna Kea/Keck/LRIS & $\sim 2650$ & $6425-7692 \AA$ & 12 & CFHT1999 \\
\hline Nov. 2-5, 2002 & Mauna Kea/Keck/LRIS & $\sim 950$ & $6250-9600 \AA$ & 29 & CFHT1999 \\
\hline Dec. $11-14,2002$ & Las Campanas/Magellan/MIKE & $\sim 11250$ & $4430-7250 \AA$ & 14 & CFHT1999 \\
\hline Mar. 9-11, 2003 & Las Campanas/Magellan/B\&C & $\sim 2600$ & $6200-7825 \AA$ & 2 & CFHT1999 \\
\hline Mar. 9-11, 2003 & Las Campanas/Magellan/B\&C & $\sim 800$ & $5000-10200 \AA$ & 3 & CFHT1999 \\
\hline Nov. $22-25,2005$ & CAHA/3.5 m/TWIN & $\sim 1100$ & $5600-10425 \AA$ & 5 & CFHT1999 \\
\hline Nov. 30-Dec. 11, 2007 & CAHA $/ 2.2 \mathrm{~m} / \mathrm{CAFOS}$ & $\sim 600$ & $6200-10350 \AA$ & 37 & $\begin{array}{c}\text { CFHT1999 \& } 1^{\circ} \times 1^{\circ} \text { Spitzer } \& \\
\text { XMM-Newton } \text { survey }\end{array}$ \\
\hline Jan. 5, 2008 & Paranal/VLT /FLAMES & $\sim 8600$ & $6438-7184 \AA$ & 40 & CFHT1999 \& $1^{\circ} \times 1^{\circ}$ Spitzer \\
\hline
\end{tabular}

Table 2. A summary of the Collinder 69 near infrared spectroscopic campaigns.

\begin{tabular}{lccccc}
\hline \hline Date & Observatory/Telescope/Instrument & $\begin{array}{c}\text { Resolution } \\
\Delta \lambda / \lambda\end{array}$ & $\begin{array}{c}\text { Wavelength } \\
\text { coverage }\end{array}$ & $\begin{array}{c}\text { Number of sources } \\
\text { observed }\end{array}$ & $\begin{array}{c}\text { Original photometric } \\
\text { survey }\end{array}$ \\
\hline Dec. 22-23, 2004 & Mauna Kea/Keck/NIRSPEC & $\sim 2000$ & $1.143-1.375 \mu \mathrm{m}$ & $4^{*}$ & CFHT1999 \\
Dec. 9, 2005 & Mauna Kea/Keck/NIRSPEC & $\sim 2000$ & $1.143-1.375 \mu \mathrm{m}$ & $9^{*}$ & CFHT1999 \\
Jan. 9-11, 2007 & La Silla/NTT /SOFI & $\sim 950$ & $0.950-2.500 \mu \mathrm{m}$ & 2 & CFHT1999 \\
Nov. 10, 2008 & Mauna Kea/Subaru /IRCS & $\sim 150$ & $1.400-2.500 \mu \mathrm{m}$ & $8^{*}$ & Subaru2006 \\
\hline
\end{tabular}

Notes. ${ }^{(*)}$ For some of the sources, the obtained $\mathrm{S} / \mathrm{N}$ was too low because of poor weather conditions to perform the analysis, hence are not listed in the tables with the results from these analyses.

\section{- Near infrared:}

1. 2MASS ( $J, H, K$ s bands) provides near-infrared data down to a limiting magnitude of $J=16.8, H=16.1$, and $K \mathrm{~s}=15.3 \mathrm{mag}\left(\sim 30 M_{\text {Jupiter }}\right.$ for a DUSTY, Chabrier et al. 2000, 5 Myr isochrone).

2. INGRID Survey ( $J, H, K$ s bands): described in Barrado y Navascués et al. (2007), the detection limit can be estimated as $J_{\text {Limit }}=21.1 \mathrm{mag}$ and the completeness limit as $J_{\text {Complete }}=19.5 \mathrm{mag}$. For a DUSTY $5 \mathrm{Myr}$ isochrone (Chabrier et al. 2000), and the distance and standard extinction for the cluster, this limit corresponds to $10 M_{\text {Jupiter }}$.

3. Omega2000 Survey ( $J, H, K$ s bands): described in Barrado y Navascués et al. (2007), the completeness limits for the survey are: $J_{\text {Complete }}=20 \mathrm{mag}, H_{\text {Complete }}=19$, and $K \mathrm{~s}_{\text {Complete }}=18$. For a DUSTY $5 \mathrm{Myr}$ isochrone (Chabrier et al. 2000), and the distance and standard extinction for the cluster, this limit corresponds to $8 M_{\text {Jupiter }}$.

- Mid infrared:

1. Spitzer mid-IR imaging (3.6, 4.5, 5.8, 8.0, and $24.0 \mu \mathrm{m})$ : described in Barrado y Navascués et al. (2007) and Morales-Calderón (2008), the completeness limits are at $[3.6]_{\text {Complete }}=16.5 \mathrm{mag},[4.5]_{\text {Complete }}=16.5$, $[5.8]_{\text {Complete }}=14.5$, and $[8.0]_{\text {Complete }}=13.75$. For cluster members, the completeness limit at $3.6 \mu \mathrm{m}$ (for a $5 \mathrm{Myr}$ isochrone by Baraffe et al. 1998) corresponds to a mass $\sim 0.04 M_{\odot}$.

- X-rays:

1. XMM-Newton observations of Collinder 69: the observations consisted of two fields, one to the east and another to the west of the bright star $\lambda$ Ori, and allowed us to study the weak-line T Tauri population of this open cluster. A list of detections was compiled, cross-matched with our previous photometric surveys, and several selection criteria were applied to derive a catalog of new candidate members (see details of the whole process in Barrado et al. 2011). This study produced a list of 66 candidates (19 new) from which 44 sources have been spectroscopically confirmed already by Dolan \& Mathieu (1999); Barrado y Navascués et al. (2004b); Sacco et al. (2008); Maxted et al. (2008), or this work (four of them have only been confirmed by us). The X-ray detected cluster sample is complete down to $\sim 0.3 M_{\odot}$, with some detections for confirmed members of mass close to $0.1 M_{\odot}$.

\section{Spectroscopic observations and data analysis}

To perform the spectral analysis, we used our own data (see Sects. 3.1 and 3.2) and the measurements from Dolan \& Mathieu (2001); Barrado y Navascués et al. (2004b); Sacco et al. (2008); Maxted et al. (2008).

In brief, Dolan \& Mathieu (2001) obtained $R \sim 20000$ spectra with a setup that allowed them to study the Li I $\lambda 6707 \AA$ and $\mathrm{H} \alpha$ lines, and a rich array of metal lines near $6450 \AA$ for precise radial velocity measurements to confirm members down to $\sim 0.3 M_{\odot}$. Strong $\mathrm{H} \alpha$ emission (indicative of accretion) has also been detected from three candidate substellar members in the very deep survey for young stars of Barrado y Navascués et al. (2004b). Finally, Sacco et al. (2008) and Maxted et al. (2008) published their results on the comparative analysis of high resolution spectra of two samples of low-mass young stars (candidate members to $\sigma$ Ori and Collinder 69 clusters). Adopting a resolution of $R \sim 16000-17000$, they studied binarity, Li absorption, $\mathrm{H} \alpha$ emission, and derived rotational velocities for candidates from Dolan \& Mathieu (1999) and Barrado y Navascués et al. (2004b) close to the brown dwarf domain.

Owing to the large amount of data that we analyzed (particularly for own our data-sets, see Tables 1 and 2 and more details in Bayo 2009), we developed an automatic procedure to perform 
the line characterization. We also studied the effect of the spectral resolution in the measurements provided by this automatic procedure (see Appendix A).

\subsection{Optical spectroscopy}

During the past seven years, our group has been granted time at different observatories to perform spectroscopic observations of the previously described candidates. These observations comprise a wide range of resolutions and wavelength coverages. In Table 1, the most relevant information about the different runs is displayed.

Except where otherwise stated, we reduced all data within the $\operatorname{IRAF}^{1}$ environment in as standard way.

When observing with the LRIS, Low Resolution Imaging Spectrometer (Keck), We used the 1200 lines/mm grating, with a scale of $0.63^{\prime \prime} /$ pixel and a resolution of $\sim 2 \AA$ (measured in a NeHe comparison lamp, $R \sim 2650$ ). The one arsec slit was used and a wavelength coverage of 6425-7692 $\AA$ was studied. During the same run, we collected low-resolution spectra with the $400 \mathrm{l} / \mathrm{mm}$ grating and also the one arcsec slit. The wavelength calibration is more accurate than $0.4 \AA$, the resolution is $6.0 \AA$ around the wavelength of $\mathrm{H} \alpha$ as measured with a $\operatorname{NeAr}$ lamp $(R \sim 950)$, and the wavelength coverage is 6250-9600 А.

With MIKE, Magellan Inamori Kyocera Echelle (Magellan), we used the configuration prior to the 2004 Red-MIKE standard configuration of R2 echelle grating, of scale $\sim 0.29^{\prime \prime} / \mathrm{pixel}$ and $1.0^{\prime \prime}$ slit. With our set-up, the spectral coverage in the red arm was 4430-7250. . To improve the final signal-to-noise ratio $(\mathrm{S} / \mathrm{N})$, we degraded the resolution by rebinning the original data during the readout to two and eight pixels in the spatial and spectral directions, respectively, achieving a resolution of $0.55 \AA(R \sim 11250)$.

For the B\&C, Boller \& Chivens spectrograph (Magellan), We used the $12001 / \mathrm{mm}$ grating and the $1.0^{\prime \prime}$ slit with a scale of $0.79^{\prime \prime} /$ pixel a resolution of $\sim 2.5 \AA$ as measured in a HeNeAr comparison lamp $(R \sim 2600)$ and a wavelength coverage from 6200 to $7825 \AA$. We also used the 300 1/mm grating (same slit as before with a resolution of $\sim 800$ ), and a wavelength coverage 5000-10200 ̊; in this way, the Magellan spectra have slightly poorer resolution and a wider spectral range.

In the FLAMES, Fibre Large Array Multi Element Spectrograph (VLT) program 080.C-0592, we used the LR6 grating with a measured resolution of $0.76 \AA$, where each fiber has an aperture of $1.2^{\prime \prime}$ on the sky and GIRAFFE has a scale of $0.3^{\prime \prime} /$ pixel in MEDUSA (the resolution is $\sim 8600$ and the wavelength range is 6438-10350 $\mathrm{A}$ ). The data reduction was performed using the GIRAFFE gir-BLDRS pipeline vers. 1.12, following the standard steps that include correction for the differences in the fiber transmission. When processed by the pipeline, the spectra are not corrected for sky background; hence, the analysis that we present was performed after subtracting a sky spectrum. This background spectrum was computed as the median of those obtained from the fibers positioned "on sky". Since the nebular emission of the region of Collinder 69 is not negligible and our sky fibers were distributed quite homogeneously, we studied the variations in the $\mathrm{H} \alpha$ nebular emission using these sky fibers to estimate the accuracy of the correction achieved. We measured a mean full width at $10 \%$ of $\sim 41 \mathrm{~km} \mathrm{~s}^{-1}$ with a

\footnotetext{
1 IRAF is distributed by the National Optical Astronomy Observatory, which is operated by the Association of Universities for Research in Astronomy, Inc. under contract to the National Science Foundation.
}

standard deviation of $\sim 3 \mathrm{~km} \mathrm{~s}^{-1}$. Therefore, the dispersion measured in different fibers translated into an added $\sim 7 \%$ uncertainty in our measurements.

At the CAFOS, Calar Alto Faint Object Spectrograph (CAHA 2.2 m telescope), We used the $R$-200 grism, with scale of $0.53^{\prime \prime} /$ pixel and a measured resolution of $\sim 11 \AA$. In addition we used the $1.6^{\prime \prime}$ slit, $R \sim 600$, with a wavelength coverage of 6200-10350 А.

At the TWIN (CAHA 3.5 m telescope) in every run, we used the T-13 grating and the $1.2^{\prime \prime}$ and $1.5^{\prime \prime}$ slits and only the data coming from the red arm were processed. The pixel scale is $0.56^{\prime \prime} /$ pixel, and we measured a resolution of $\sim 6 \AA(R \sim 1100$ and wavelength coverage from 5600 to $10400 \AA$ ).

\subsection{Near-infrared spectroscopy}

For the SOFI, Son of ISAAC (NTT) program 078.C-0124, we used two low-resolution grisms with the $0.6^{\prime \prime}$ slit to roughly cover the JHK bands on a Hawaii HgCdTe $1024 \times 1024$ detector with a plate scale of $0.292^{\prime \prime} /$ pix. The blue grism covers $0.95-1.63 \mu \mathrm{m}$ and the red grism the range $1.53-2.52 \mu \mathrm{m}$. The corresponding spectral resolutions were 930 and 980 respectively. The telescope was nodded along the slit between two positions following the usual ABBA pattern.

In addition to the science targets, we observed several "telluric standards" (A0V objects at similar airmasses) to remove telluric water absorption bands as described by Vacca et al. (2003), and to estimate the instrumental response. A Xe arc lamp was used for the wavelength calibration (consistent with the $\mathrm{OH}$ airglow calibration) with an accuracy of $1.2 \AA$ for the blue grism, and $2 \AA$ for the red one.

Using NIRSPEC, the Near InfRared echelle SPECtrograph (Keck), in both campaigns we used Nirspec-3 with the $0.57^{\prime \prime}$ slit, covering the $1.143-1.375 \mu \mathrm{m}$ wavelength region. The corresponding spectral resolution is $\sim 2000$ and the pixel scale is $0.18^{\prime \prime} /$ pixel. The classical ABBA pattern was again followed and telluric A0V standards were observed. In this case, the data reduction process was carried out using the IDL-based software REDSPEC, which performs the standard steps in a pseudoautomatic manner where some interaction with the user is required.

With IRCS, the InfraRed Camera and Spectrograph at Subaru, we used the "Grism HK" covering a wavelength range of $1.4-2.5 \mu \mathrm{m}$ at a very low resolution of $\sim 150$ for a $0.3^{\prime \prime}$ slit and the 52 mas scale. The spectra of the science targets, spectral templates and A0V standards were obtained following an ABBA pattern and the reduction of the data was performed using IRAF in a standard manner.

\section{Temperature scale for young $M$ very low-mass stars and brown dwarfs}

\subsection{Spectral typing}

Depending on the expected nature of the sources themselves and the characteristics of the available spectra, we adopted different approaches to deriving spectral types for our candidates:

For the Optical spectra, We considered two groups according to the effective temperature derived from the spectral energy distribution (SED) fit (see Sect. 4.2): "warm" ( $T_{\text {eff }} \gtrsim 4000 \mathrm{~K}$, about M0 spectral type) and "cool" ( $\left.T_{\text {eff }} \lesssim 4000 \mathrm{~K}\right)$ sources.

For the warmer part of the sample (a fraction of the XMM candidates), we compared our optical (low resolution, CAFOS) 

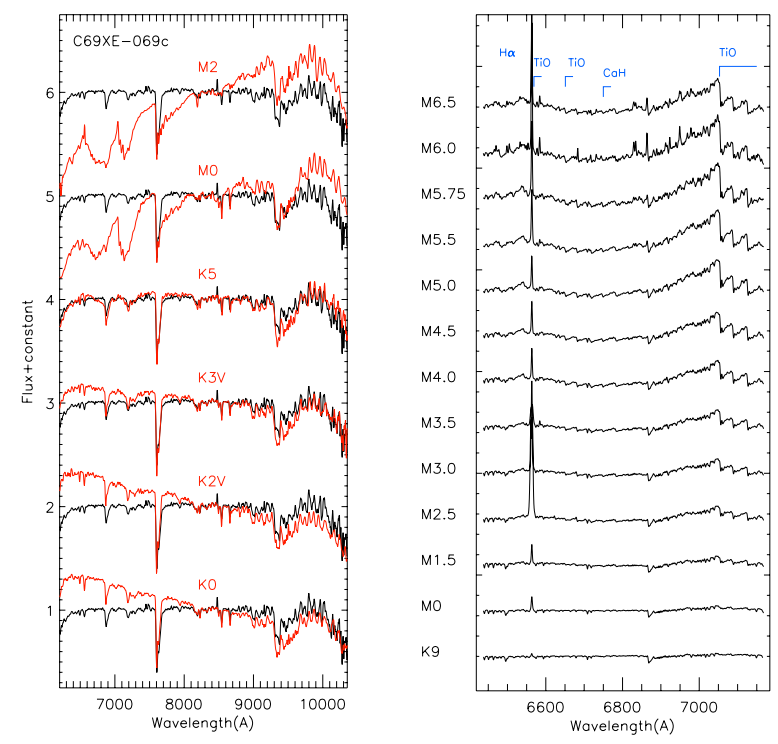

Fig. 2. Left: spectral type determination of one XMM candidate member by comparison to templates obtained with the same instrumental set-up (CAFOS). The "science spectra" are displayed in black and the templates (labelled according to the spectral type) in red. Right: spectral sequence derived for candidate members of Collinder 69 observed with FLAMES. Note the intense $\mathrm{H} \alpha$ emission and strengthening of the typical $\mathrm{TiO}$ and $\mathrm{CaH}$ bands with the spectral type.

spectra with templates (obtained with the same configuration during the same campaigns) from Taurus members and field dwarfs within a spectral type range from G0 to M0 (the comparison spectra were reddened to the previously mentioned average low extinction, $A_{v} \sim 0.36$ mag estimated by Duerr et al. 1982 for Collinder 69). We normalized both the science spectra and the templates at the same wavelength and using a simple $\chi^{2}$ minimization decided which template reproduced the science data most accurately. We did not use any specific temperaturesensitive lines because our resolution did not allow us to fit anything other than the continuum shape. As an example, in Fig. 2 we show an example of this comparison for a $\mathrm{K}$ candidate member.

The majority of our candidates belongs to the colder sample, in principle with $T_{\text {eff }}$ consistent with $\mathrm{M}$ spectral types. M-dwarfs are characterized spectroscopically by the presence of molecular bands of titanium and vanadium oxide (TiO, VO). These bands reach their maximum strength around M7, and then become weaker for cooler temperatures because of $\mathrm{Ti}$ and $\mathrm{V}$ condensation onto dust grains. Among the atomic lines, the Ca II triplet is much weaker than in M supergiant spectra, but the Na I and K I doublets are both stronger, since they are gravity dependent.

Several spectral ratios or indices (quantifying different band strengths) have been proposed in the literature to derive spectral types for these late-type stars. Some are based on the relative depths of the afore mentioned molecular bands (see for example Reid et al. 1995; Cruz \& Reid 2002 and references therein); others are based on measuring the slope of the pseudocontinuum (for example PC3 and PC6 from Martin et al. 1996, and Martín et al. 1999).

We used different combinations of these indices (depending on the resolution and wavelength coverage of the spectra) to classify our M-like candidates. In Fig. 2, we show an example of a spectral sequence obtained applying these indices to
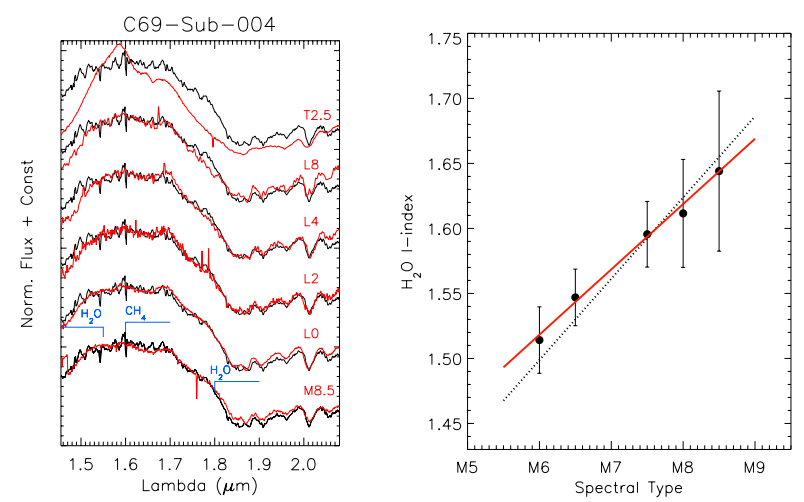

Fig. 3. Left: infra-red spectral type determination for a candidate member observed with Subaru. The templates used for comparison (red spectra with corresponding spectral type label) were also obtained with Subaru and correspond to known $\mathrm{L}$ and $\mathrm{T}$ dwarfs from Geballe et al. (2002). A visual comparison with the spectra obtained by Lodieu et al. (2008) of Upper Sco members also suggests that a L spectral type is a closer match especially based on the water bands and the "peaky" structure $\sim 1.7 \mu \mathrm{m}$. Right: our own re-calibration of the reddening independent $I_{\mathrm{H}_{2} \mathrm{O}}$ index from Comerón et al. (2000). The dotted black line represents the linear relation found by Comerón et al. (2000), while the red line is the best linear fit to our data and is the relation that we have used to estimate the spectral types of our $\mathrm{M}$ candidates.

13 candidates observed with FLAMES. This combination of indices should provide us with a spectral classification of $\sim 0.5 \mathrm{sub}$ class accuracy.

We now consider our Near-infrared spectra. Owing to their nature, the reddest-coldest candidates of our sample (late M, L, and even $\mathrm{T}$ spectral types according to the SED fitted temperatures) more carefully can be studied in the infrared than the optical. As in the optical case, we performed the spectral classification either using spectral indices (for the two $\mathrm{M}$ candidates, see right panel of Fig. 3) or by comparison with templates (for the $\mathrm{L}$ and $\mathrm{T}$ candidates, see left panel of Fig. 3).

The near-infrared spectra of $\mathrm{M}$ dwarfs are dominated by deep broad absorption bands of $\mathrm{H}_{2} \mathrm{O}$ (particularly at 1.4 and $1.85 \mu \mathrm{m}$ ). That water vapour in the Earth's atmosphere also contributes with a substantial absorption at these wavelengths hampers the analysis of these bands to some extent. However, the higher temperatures in the stellar atmospheres mean that the associated steam bands are broader than the terrestrial absorption, hence the wings are accessible for measurement and analysis.

Apart from these water bands, other molecular bands such as the $\mathrm{CO}$ (at $2.29 \mu \mathrm{m}$ ), $\mathrm{FeH}$ (at $0.99 \mu \mathrm{m}$ ), and VO (at $1.2 \mu \mathrm{m}$ ) also scale with temperature (Jones et al. 1994). Nevertheless, these changes are not as dramatic as those observed in the water bands and, therefore, we focus on the former to derive the spectral types.

Comerón et al. (2000) defined a reddening independent index, $I_{\mathrm{H}_{2} \mathrm{O}}$, to measure the depth of the wings of the water band of late M dwarfs centered near $1.9 \mu \mathrm{m}$. Gómez \& Persi (2002) tested this method by comparing the estimated spectral types with the $Q$ index defined by Wilking et al. (1999) and obtained excellent agreement (see Fig. 8 in their paper). This $Q$ index is also reddening independent and was defined to characterize the strength of the $1.7-2.1 \mu \mathrm{m}$ and $\geq 2.4 \mu \mathrm{m}$ water absorption bands.

Owing to the wavelength coverage of our observations, we only used the $I_{\mathrm{H}_{2} \mathrm{O}}$ index to estimate infrared spectral types of the candidates. In the right panel of Fig. 3 (dotted line), we show the linear relation proposed by Comerón et al. (2000) between 
this index and the spectral type (obtained for confirmed members of Chamaeleon I, $\sim 1$ Myr old). We re-calibrated this relation using a sample of well-known field M-dwarfs (filled black circles with error bars illustrating the dispersion in the measurements for $\sim 3$ objects per spectral type) obtaining a very similar slope (red line). With this comparison, we see how, in the M5.5M9 spectral range, the $I_{\mathrm{H}_{2} \mathrm{O}}$ index is insensitive to the known age dependency on the water bands. Keeping this caveat of the age uncertainty in mind, this relationship allows us to estimate spectral types to within 1.5 sub-types. For the two objects where we used this method, we also had optical spectra. In both cases, the infrared spectral type is colder than the optical one but within the error bars. This might be indeed a result of the age dependency of the $I_{\mathrm{H}_{2} \mathrm{O}}$ index. Finally, both sources were classified as diskless based on their IRAC photometry, hence we can exclude the possibility that some excesses caused by the disk is affecting our classification.

Both the optical and near-infrared spectral types derived by us are listed in Table 6.

\subsection{SED fitting}

As can be inferred from Sect. 2, the photometric surveys provided us with a very large but inhomogeneous dataset. To analyze these data in a homogeneous and automatic manner, we used the tool VOSA (Bayo et al. 2008).

In short, the user provides VOSA with a table with photometry and the tool performs the following tasks in an almost automatic way (very little interaction is needed): it enlarges the wavelength coverage by looking for counterparts in different (VO-compliant) catalogues, it compiles the SEDs for all the sources, and it performs the SED fit using models (Hauschildt et al. 1999; Allard et al. 2001; Chabrier et al. 2000; Castelli et al. 1997) of stellar and substellar photospheres (effective temperature, surface gravity, and metallicity as free parameters), it uses those models to obtain a multi-color bolometric correction and, finally interpolates among isochrones and evolutionary tracks (Baraffe et al. 1998, 2002; Chabrier et al. 2000; Baraffe et al. 2003) to derive ages and masses.

In addition to the photometric data, the user has to provide VOSA with the estimated distance to each source and the extinction affecting the line of sight. In the case of Collinder 69, this does not represent any extra complication, since members should be located approximately at the distance derived for the cluster (400 pc, Murdin \& Penston 1977) and we benefit from the low extinction $\left(A_{v} \sim 0.36 \mathrm{mag}\right.$, Duerr et al. 1982) affecting this region.

The parameters derived in this fashion are listed in Table 6. The relation between this VO methodology and other classical ones can be found in Bayo et al. (2008). In particular, some of the possible caveats to take into account in the determination of $T_{\text {eff }}, L_{\text {bol }}$ and Mass via SED fit are:

- Blue excess in the SED caused by accretion. This aspect is discussed in Paper II, where we study in detail the veiling in the objects with the strongest $\mathrm{H} \alpha$ emission and demonstrate that for these cool objects veiling is hardly noticeable, hence the determination of $T_{\mathrm{eff}}, L_{\mathrm{bol}}$, and mass via SED fit is insensitive to it.

- Red excess in the SED caused by a circumstellar disk. The impact of circumstellar disks on our SED fits is discussed in Bayo et al. (2008), where we showed that only edge-on disks above a certain mass (below which, the disk is not massive enough to produce an effect in the photospheric part of the

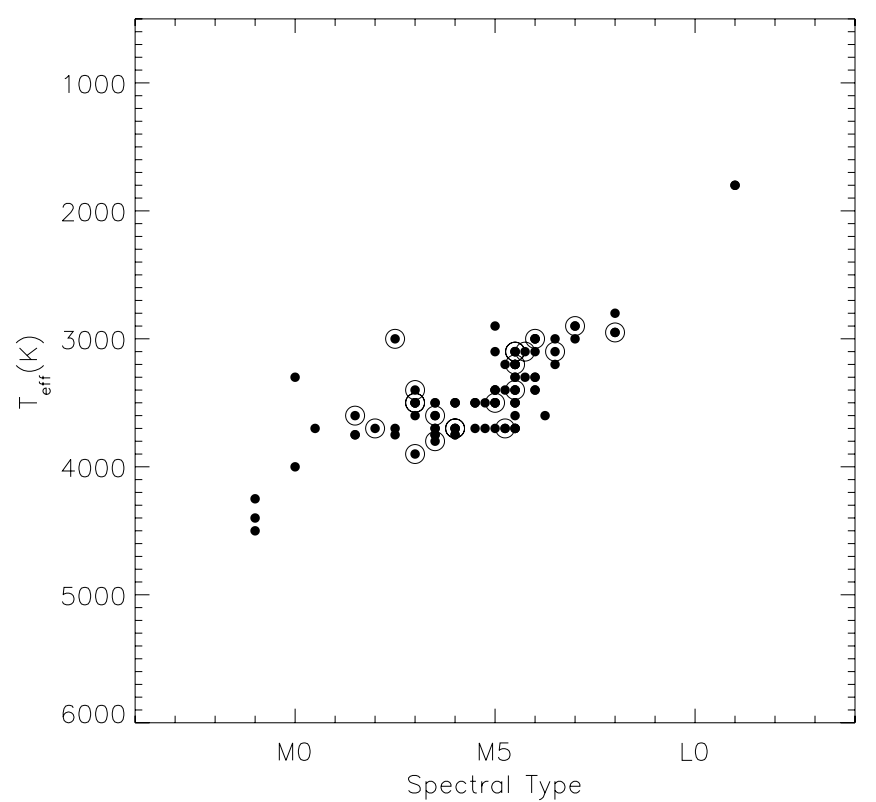

Fig. 4. Comparison between the spectral types determined from spectra and the effective temperature estimated via an SED fit. We only display the spectroscopically confirmed members here, highlighting the sources that harbour disks with large circumferences. It is clear that no systematic differences in $T_{\text {eff }}$ estimation can be attributed to the presence of disks.

SED) introduce a bias in our parameter estimations. When this is the case, our methodology will underestimate both the effective temperature and the bolometric luminosity of the central object. From the SED shape of the confirmed members (no infrared peak higher than the photospheric one), we can infer that we do not have edge-on disks in our sample. In any case, to illustrate this point more clearly, in Fig. 4 we plot the estimated spectral type versus effective temperature. While the latter could in principle be affected by the disk, the former should not since we use blue features of the optical spectra to estimate the spectral type. In the figure, we have highlighted the objects that harbour disks by plotting large circles around them. We can see how the dispersion in effective temperature among the objects of the same spectral type does not correlate with the presence of disks.

- Gray excess in the SED due to multiplicity. To study the effects of possible unresolved multiple systems, we performed a simple exercise considering two cases: a similar mass ratio and an "extreme" one. In both cases, we compiled simulated SEDs by adding the fluxes of two pairs of confirmed members and performed the fit in the composite SED. As expected, when the components have similar characteristics (in this particular case, M4 spectral type and diskless sources); the derived effective temperature does not change, but VOSA over-estimates the mass and $L_{\mathrm{bol}}$ of the individual components (and of course, underestimates the age). However, for the most extreme case that we could build (class III sources but with 5000 and $2800 \mathrm{~K}$ effective temperatures), the resulting $T_{\text {eff }}$ is very close to that of the hottest component $(4700 \mathrm{~K})$ and the $L_{\text {bol }}$ higher than that estimated for the hottest component, but still almost within the error-bars. Thus, in this case again, the most significant effect to keep in mind is that the age of the system would be underestimated. In any case, according to Sacco et al. (2008) and Maxted et al. (2008), the short-period binarity fraction in Collinder 69 is as low as $\sim 10 \%$ (unlike 
Table 3. Temperature scale derived from our spectroscopic data of Collinder 69.

\begin{tabular}{lc}
\hline \hline Spectral type & Effective temperature \\
\hline M0 & $4000 \mathrm{~K}$ \\
M1.5 & $3750 \mathrm{~K}$ \\
M2.5 & $3600 \mathrm{~K}$ \\
M3.5 & $3500 \mathrm{~K}$ \\
M4 & $3500 \mathrm{~K}$ \\
M4.5 & $3300 \mathrm{~K}$ \\
M5 & $3200 \mathrm{~K}$ \\
M5.5 & $3260 \mathrm{~K}$ \\
M6 & $3100 \mathrm{~K}$ \\
M6.5 & $3050 \mathrm{~K}$ \\
M7 & $3000 \mathrm{~K}$ \\
M8 & $2700 \mathrm{~K}$ \\
\hline
\end{tabular}

the higher $\sim 30 \%$ reported for field M dwarfs by Reid \& Gizis 1997); hence, although for the individual targets we should keep these possible biases in mind, the general conclusions about the cluster as a whole should be unaffected by binarity.

\subsection{Effective temperature scale}

Several temperature scales for $\mathrm{M}$ dwarfs are already available in the literature (Bessell et al. 1998; Luhman 1999; Leggett et al. 2000; Luhman et al. 2003). However, in many cases, the sample of objects for which this scale is derived lacks homogeneity and/or is based on small numbers statistics. As an example, the set of objects studied sometimes comprises sources with different ages or environments, hence the possible effects of those factors cannot be addressed.

With this in mind, using the determinations of spectral types and effective temperatures from the previous two subsections, we derived our own temperature scale. We took advantage of the homogeneity of our determination of both spectral types and effective temperature and that we studied objects of roughly the same age, which were born in the same environment (an environment with the practical advantage of low extinction affecting the observational data). To derive the temperature scale, we started by defining a "clean" sample (see Table 3 and Fig. 5). This "clean" sample is composed of $\sim 30$ sources fulfilling every one of the next criteria: membership confirmed through lithium absorption (see next section), SED with no infrared excess whatsoever (to avoid problems in $T_{\text {eff }}$ determination implied by edgeon disks with VOSA, see Sect. 4.2 and Bayo et al. 2008), optical spectral type derived between M0 and M8 (see Sect. 4.1), no signs of variability or binarity, and estimated age according to the HR diagram between 1 and $10 \mathrm{Myr}$ (see Fig. 11).

The temperature scale determined in this manner is shown in Table 3. In Fig. 5, we compare our scale with others from the literature (Luhman 1999; Bessell et al. 1998; Luhman et al. 2003). We find a good agreement with the scale derived by Luhman (1999) for a sample of Taurus members (1-3 Myr, slightly younger than Collinder 69) but systematically higher temperatures than those proposed by Bessell et al. (1998) and Luhman et al. (2003). With respect to the Bessell et al. (1998) scale, we probably see only the effect of the difference in age from the two samples of objects for which the scales are derived.

However, the scale presented by Luhman et al. (2003) corresponds to an update to the one derived in Luhman (1999), but in this case for the young cluster IC 348 ( $~ 2$ Myr old). Therefore, the differences between the two scales in this case should not be related to an aging effect. In any case, these differences are

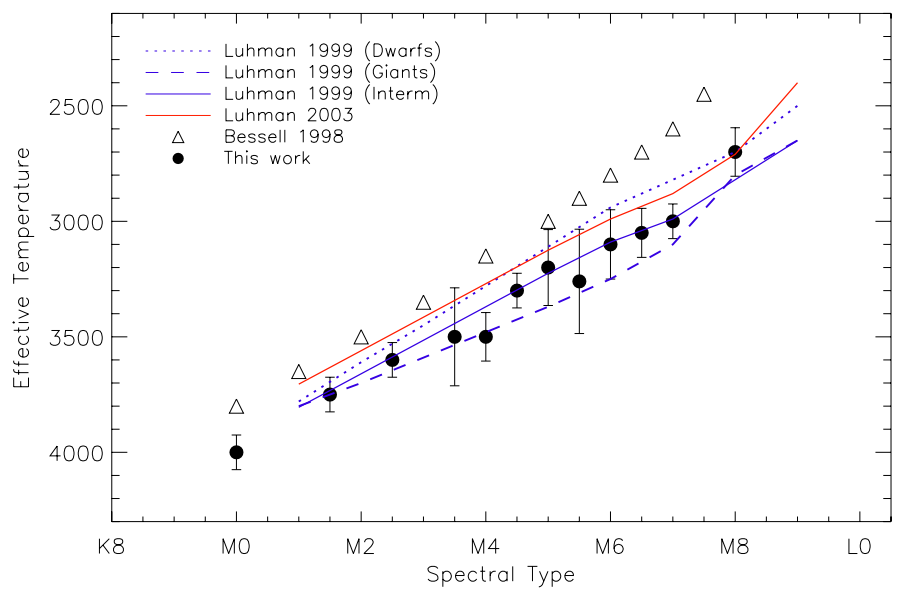

Fig. 5. Temperature scale derived for the confirmed very low mass $\mathrm{M}$ star and brown dwarf members of Collinder 69. With different symbols and line styles, we also display scales previously reported in the literature.

insignificant taking into account the dispersion in estimated $T_{\text {eff }}$ that we see for an individual subclass (especially between M3.5 and M6).

In this same line, we must note that the dispersion previously mentioned, seems to be of the same order as the differences found by Luhman (1999) between dwarfs and giants (while none of our confirmed members can have a low luminosity class according to our study). This dispersion seems to be larger for specific spectral types and might provide a measure of some physical properties of the atmospheres of objects within this range of temperatures. It could be related to dust settling, although the temperature range seems to be too large for this phenomenon to happen. It could be related to metallicity, but, to the best of our knowledge, there are no detailed metallicity studies in this cluster. In any case, our "clean" sample is not well enough sampled to allow us to derive any conclusion.

\section{Confirming membership}

To confirm the membership of our candidates we have used different diagnostics (all of them based on alkali absorption lines) depending mainly on the resolution of the spectra.

\subsection{Lithium absorption at $6708 \AA$}

Above $\sim 0.065 M_{\odot}$ (less massive objects cannot develop the necessary temperature in their cores), lithium acts as an age indicator because the time it takes for the core to reach $3.0 \times 10^{6} \mathrm{~K}$ is a sensitive function of mass (Basri 1997). Very low mass stars and brown dwarfs down to this mass limit are fully convective, hence once the core temperature exceeds the necessary limit, the entire lithium content of the star should be exhausted rapidly leading to an observable change in the photospheric lithium abundance.Theoretical models make specific predictions about the time evolution of this lithium depletion boundary. For example, Ventura et al. (1998) predict that at ages 30, 70, and $140 \mathrm{Myr}$, the lithium depletion edge should occur at $0.17,0.09$, and $0.07 M_{\odot}$, respectively. Other models by Chabrier \& Baraffe (1997) and Burrows et al. (1997) make similar predictions about the variation in this lithium depletion boundary with age. Bildsten et al. (1997) and others have indeed argued that the age for an open cluster derived in this manner should be more accurate than 


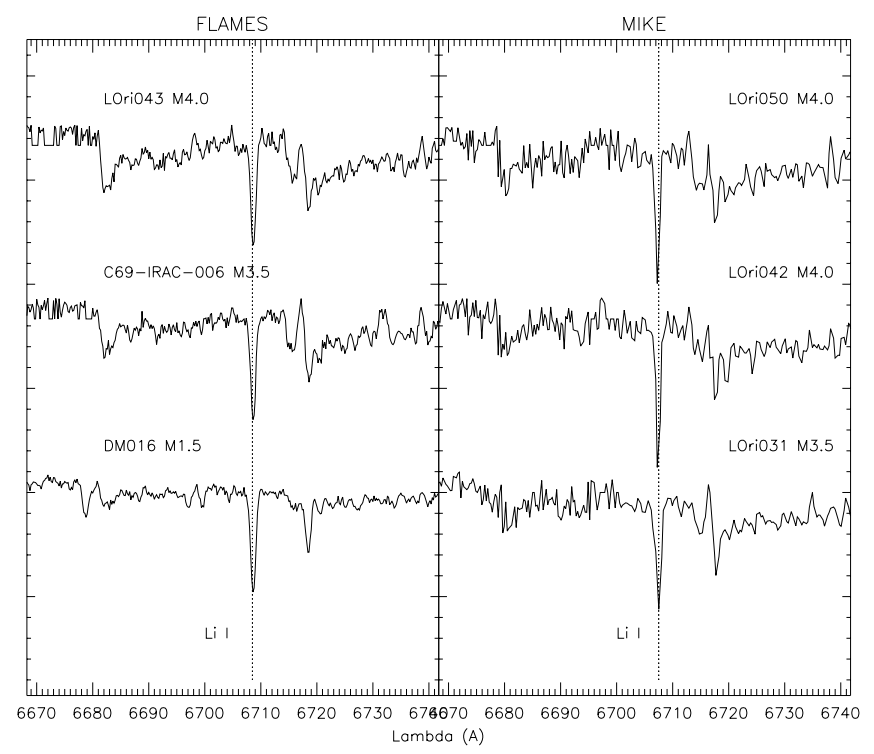

Fig. 6. Detail around Li I $\lambda 6708 \AA$ for eight confirmed members with different spectral types. The two panels correspond to two different campaigns: Magellan/MIKE echelle spectra (only the order corresponding to this line), and FLAMES multi-fiber spectra.

those found by any other method (Stauffer et al. 1998, 1999; Barrado y Navascués et al. 1999; Jeffries et al. 2003; Barrado y Navascués et al. 2004b; Jeffries \& Oliveira 2005; Jeffries et al. 2009).

Owing to the intrinsic weakness of the lithium line, we have only been able to use it as a youth indicator for the sources observed with a high $\mathrm{S} / \mathrm{N}$ (especially in the lower resolution campaigns) and down to a certain spectral resolution (see Appendix A). Whenever these two factors were favorable, the lithium presence (and absence) was considered the main component to assess membership in Table 6. As an example, Fig. 6 shows two objects with clear Li I absorption in spectra taken with different instrumental set-ups.

To illustrate the presence of $\mathrm{Li} \mathrm{I}$ in absorption as a youth indicator, Fig. 7 shows the measured lithium equivalent width versus the spectral type of the (newly confirmed) members to Collinder 69. In the middle panel, we included the data from Dolan \& Mathieu (1999, 2001); Sacco et al. (2008), along with the measurements from this work. Whenever more than one measurement of the $\mathrm{Li}$ equivalent width was available, we have displayed the average value. For comparison, in the bottom panel of the same figure, we have included data corresponding to a similar age cluster, $\sigma$ Orionis, as well as (in all the panels) an upper envelope of the values measured in older clusters (see caption of the figure). We have also plotted the theoretical equivalent widths from Zapatero Osorio et al. (2002) for both $\log g=4.0$ and $\log g=4.5$ and initial cosmic abundance $(A(\mathrm{Li})=3.1)$.

The scatter in the Li I equivalent widths is considerable for all spectral types included in the figure, and even larger in the $\mathrm{M}$ domain. This is a well-known trend for which there is not yet any clear explanation (see Barrado y Navascués et al. 2001a; Zapatero Osorio et al. 2002, and more recently da Silva et al. 2009 and references therein). The dispersion could be ascribed to a variability in the $\mathrm{Li}$ I line as a consequence of stellar activity, different mixing processes, presence or absence of circumstellar disks, binarity, or different rotation rates from star to star. Baraffe $\&$ Chabrier (2010) tried to explain this dispersion in terms of the early accretion history that could lead to a dispersion in the $\mathrm{Li}$ abundances of young low mass stars and brown dwarfs. In

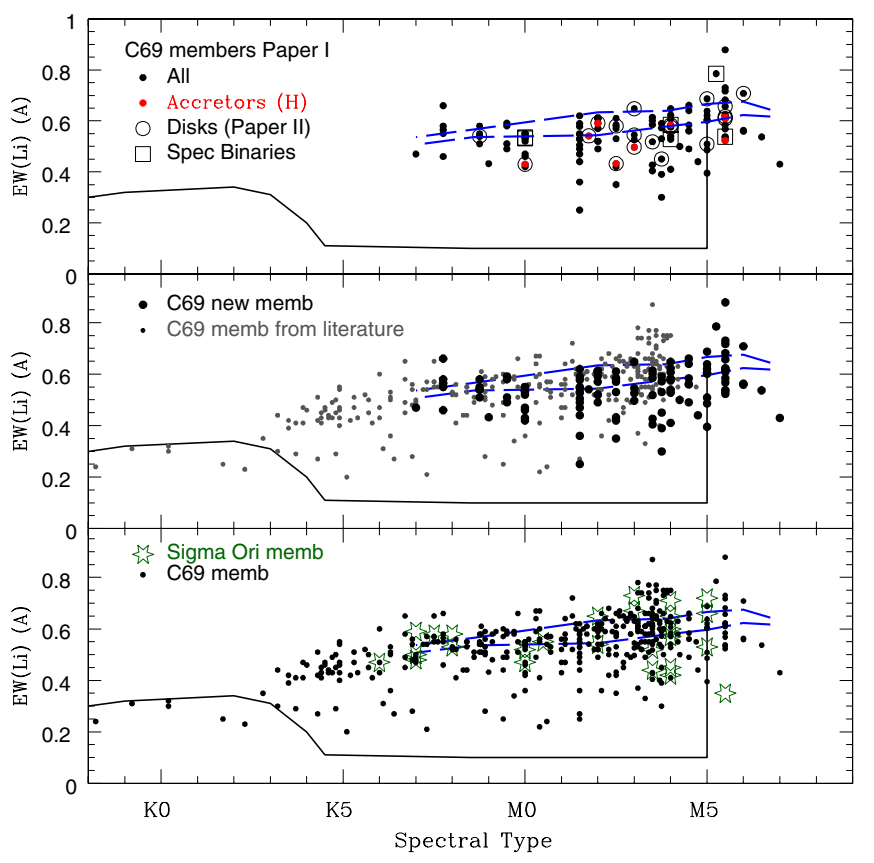

Fig. 7. Lithium equivalent width ( $E W$, in $\AA$ ) versus the spectral type for different sets of spectroscopically confirmed members of Collinder 69. Top: new members reported in this work where accretors (based on the $\mathrm{H} \alpha$ emission, see Paper II for details) are highlighted in red, and class II sources (based on IRAC data, see Barrado y Navascués et al. 2007 and Paper II) and spectroscopic binaries (from Sacco et al. 2008 and Maxted et al. 2008) are surrounded by open circles and squares, respectively. Middle: new members are shown as filled black circles, while members from the literature (Dolan \& Mathieu 1999, 2001; Barrado y Navascués et al. 2004b, 2007) are shown as smaller grey circles. Bottom: comparison of Collinder 69 members (joining the samples from the middle panel), displayed as filled black dots, with Sigma Orionis low mass stars from Zapatero Osorio et al. (2002), represented with star symbols. In all the panels, the solid line traces the upper envelope of the values measured in older clusters such as IC 2391, IC 2602, the Pleiades, and M 35; and the short-dashed blue lines corresponds to the cosmic abundances $-A(\mathrm{Li})=3.1-$ from gravities of $\log g=4.5$ and 4.0, respectively (curves of growth from Zapatero Osorio et al. 2002).

the top panel of Fig. 7, we have plotted red dots on top of the sources in our sample classified as accretors (based on the $\mathrm{H} \alpha$ equivalent width, see Bayo et al. 2011b, hereafter Paper II, for details), large open circles around those showing infrared excess, and large open squares for those sources classified as binaries by either Sacco et al. (2008) or Maxted et al. (2008). It is quite clear that none of these particular sets of objects have distinct positions in the diagram. Therefore, the cause of the scatter observed in these measurements remains unknown.

On the basis of this scatter, da Silva et al. (2009) highlighted the possible problems of using $\mathrm{Li}$ to date individual stars. We tried to quantify whether this can be a problem in our case, and we found that in all cases (16 sources) where membership was confirmed by either Dolan \& Mathieu (2001) or this work based on $\mathrm{Li}$, and where Maxted et al. (2008) had studied the radial velocity, membership had also been confirmed by Maxted et al. (2008). We note, however, that we are unable to measure the opposite effect, since for the only two objects that we discarded as members based merely on their lithium measurement (LOri011 and LOri012), there is no radial velocity measurement to compare with. In any case, for very young associations such as this one, and for the estimated spectral types of these two objects 
Table 4. Properties of the sources showing lithium $E W$ variability.

\begin{tabular}{|c|c|c|c|c|c|c|c|c|}
\hline Object & SpT & INS & Binarity ${ }^{1}$ & IR Class & $T_{\mathrm{eff}^{2}}{ }^{2}$ & $v \operatorname{sinin}^{3}$ & $\mathrm{H} \alpha$ Vble $^{4}$ & X-ray ${ }^{5}$ \\
\hline LOri013 & M3.5 & (8) & & III & 3750 & - & - & $\mathrm{Y}$ \\
\hline LOri045 & M3 & (8) (6) & & III & 3500 & $<17$ & Y & $\mathrm{N}$ \\
\hline LOri057 & M5.5 & (3) & & III & 3700 & $<17.0$ & $\mathrm{~N}$ & $\mathrm{~N}$ \\
\hline LOri063 & M4.0 & (3) & & II & 3700 & $<18.2$ & Y & $\mathrm{N}$ \\
\hline LOri068 & M4.5 M5.0 & (1) (3) & & III & 3700.0 & $<17.0$ & Y & $\mathrm{N}$ \\
\hline LOri075 & M5.0 M5.5 & (2) (8) (3) & SB1 (M08) & III & 3400 & $61.3_{-4.9}^{+11.5}$ & Y & $\mathrm{N}$ \\
\hline LOri088 & M5.5 & (8) & & III & 3200 & $<17.0$ & Y & $\mathrm{N}$ \\
\hline LOri094 & M5.5 & (8) (1) & & III & 3200.0 & $54.8_{-8.2}^{+5.5}$ & Y & $\mathrm{N}$ \\
\hline LOri106 & M5.5 & (1) & & II & 3200 & $<17.0$ & $\mathrm{Y}$ & $\mathrm{N}$ \\
\hline
\end{tabular}

Notes. (Sacco et al. 2008; Dolan \& Mathieu 1999, 2001 values compared to the values derived in this work, third column.) Note that there is no bias in terms of the resolution of the spectra (as we also proved with the exercise of Appendix A). The instrument code is the same as in Table 6. (1) According to Sacco et al. (2008) (S08) or Maxted et al. (2008) (M08). (2) Derived with VOSA. ${ }^{(3)}$ From Sacco et al. (2008). ${ }^{(4)}$ Comparing the measurements of this work (see Paper II) and/or with those of Sacco et al. (2008). ${ }^{(5)}$ Sources detected in our XMM-Newton survey (see Sect. 2.1).

(K8 and K9), even with scatter, lithium should be detectable (see Fig. 2 of da Silva et al. 2009, for more details).

In addition to the dispersion in the lithium EWs among sources with different temperatures, for some objects we observed some variability in this line when comparing our data with the values measured by Sacco et al. (2008) or Dolan \& Mathieu (1999, 2001) (see also Sect. 5 on Paper II). We display our measurements with theirs in Fig. 8. Most of our values appear to be in good agreement (within the error bars) with those of the literature. However, for the sources listed in Table 4, the differences cannot be explained in terms of the uncertainty in the measurements. Most of these objects show features typical of active stars (such as X-ray emission or $\mathrm{H} \alpha$ variability). Neuhaeuser et al. (1998) monitored the young star Par 1724 finding a variable lithium equivalent width consistent with rotational modulation. In any case, we have only two measurements per source, and the sample is too small to reach any conclusion.

\subsection{Sodium and potassium absorptions}

In terms of the lower resolution sample of sources, other alkali lines (more prominent than Li I) are known to be gravity sensitive in M-type stars. This is the case for some sodium and potassium doublets: K I at 7665 and $7700 \AA$ and Na I at 8184 and 8195.5 in the optical and K I at 1.169 and $1.177 \mu \mathrm{m}$ and 1.244 and $1.253 \mu \mathrm{m}$ in the near-infrared (see Martin et al. 1996; Schiavon et al. 1997). The surface gravity of members of Collinder 69 for this range of temperatures is expected to be $\log (g)=3-4$ (Baraffe et al. 1998), whereas a typical M-type giant should have $\log (g) \sim 2$ and an older main-sequence dwarf $\log (g) \sim 5$. Thus, the equivalent width of these doublets can be used to identify (and discard as members) background giants and foreground dwarfs from our sample.

To illustrate these differences, in Fig. 12, we compare the $\mathrm{Na}$ I doublet of candidate sources with those of field dwarfs of the same spectral types.

Owing to the inhomogeneous nature of our data, we had to chose the most suitable lines to compare with templates or models depending on the resolution and the wavelength coverage of each campaign. As an example, for the medium resolution LRIS sample, we were only able to study the bluest component of the optical K I doublet (the wavelength coverage of the instrumental set-up did not reach the other component). We note that the $E W$ measurement of this component in templates has a very strong dependence on the luminosity class, hence we propose that our estimated values listed in Table 6 are reliable.

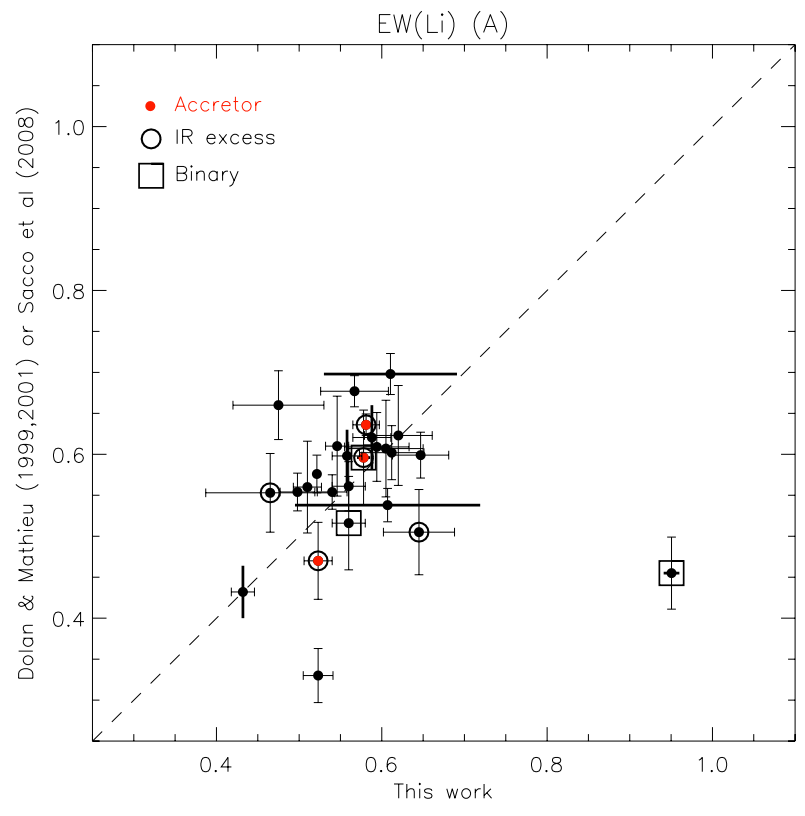

Fig. 8. Lithium equivalent width listed in Sacco et al. (2008) or Dolan \& Mathieu $(1999,2001)$ versus the values measured in this work, in $\AA$. We indicate the special sources in red (accretors), or surround them with either a circumference (objects showing infrared excess) or a square (binary systems according to Sacco et al. 2008; Maxted et al. 2008). Vertical or horizontal solid lines represent the range in variation when more than one measurement exists for one of the axes.

As mentioned before, we have also used both templates and models (Allard et al. 2003) to assess membership. In Fig. 9, we show the cases of LOri135 and LOri146, which have estimated temperatures of $3000 \mathrm{~K}$ and $2800 \mathrm{~K}$ respectively. We have normalized the science spectra and also models with those temperatures and different values of $\log (g)$. Even though the signal to noise ratio was not too high, it is very clear that these sources must have a value of $\log (g)$ that is lower than 4.0 (and higher than giant-like values, where these doublets are barely detectable).

In Fig. 10, we have plotted the effective temperature (in the range where the method is applicable, see Sect. 5.3) of members and rejected candidates to Collinder 69 against their measured $\mathrm{Na}$ I $E W$ (the 18200 doublet). We have also included the measurements from Maxted et al. (2008) and highlighted in red those sources showing X-ray emission and/or sources that are classified as active accretors in Paper II. The source LOri1 15 is "peculiar" (see later in the text) and is marked as well. As we did with 


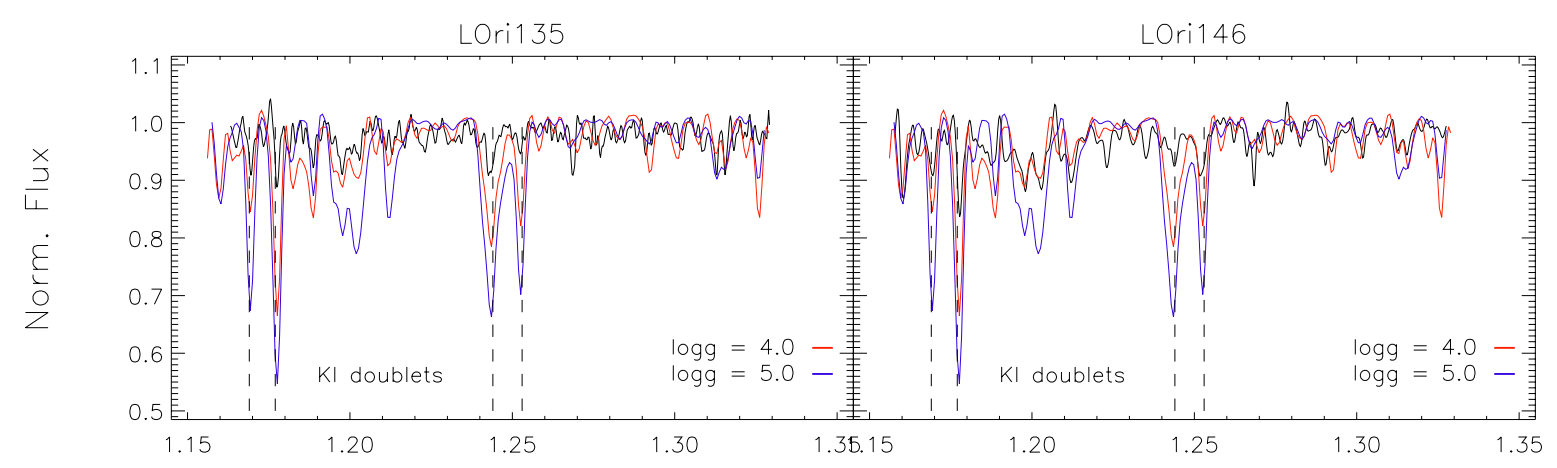

Fig. 9. Example of the surface gravity estimation for two NIRSPEC spectra by comparison with theoretical models.

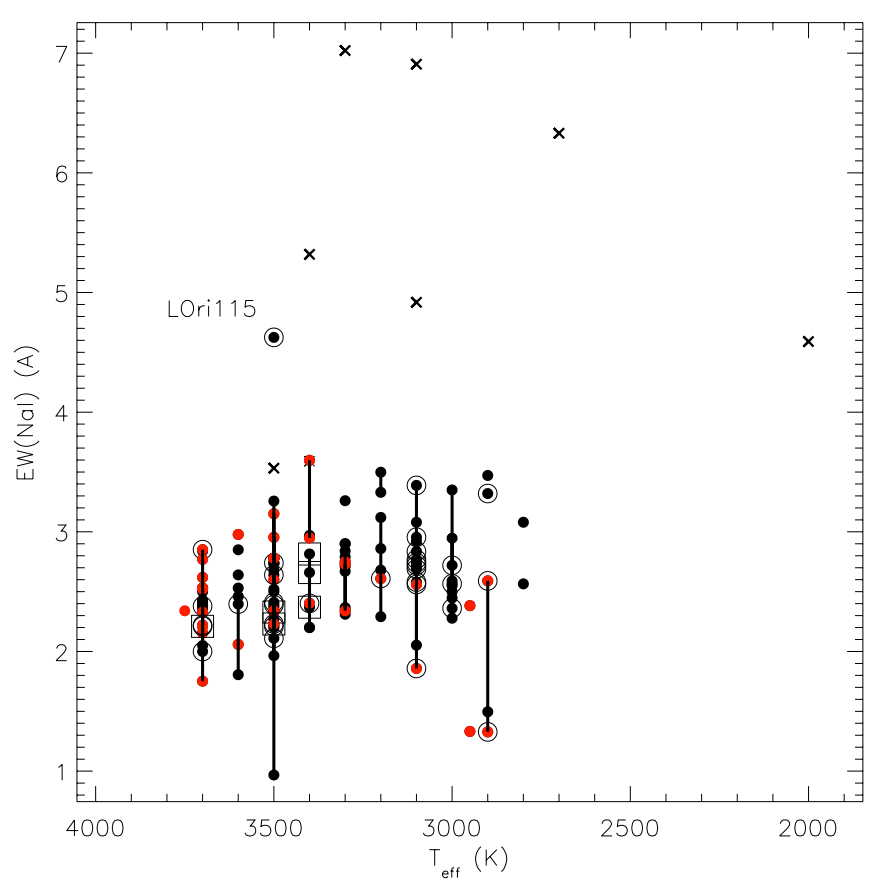

Fig. 10. $T_{\text {eff }}$ versus $E W(\mathrm{NaI})$ for members and non-members of Collinder 69. Rejected candidates are displayed as crosses, confirmed members as solid dots, where red ones highlight sources classified as active accretors and/or detected in our X-ray survey. Large open circles and squares surround sources showing infrared excess or classified as binaries in either Sacco et al. (2008) or Maxted et al. (2008). For those sources showing large variability in the measured $\mathrm{Na} I$ equivalent widths (measurements from Maxted et al. 2008 and this work), we have included vertical bars connecting those measurements. We note the unusual position of LOri115 (discussed in Sect. 5.3).

the Li I measurements, we compared the values measured by us with those from Maxted et al. (2008) for the sources in common. We find that $\sim 35 \%$ of the common sources show Na I variability (differences that are not compatible within the error bars for both measurements). The majority of these sources are either class II sources or X-ray emitters and therefore this variability must be mainly related to either activity or accretion processes.

This diagram may also suggest that in the substellar domain (the boundary is located at $T_{\text {eff }} \sim 3150 \mathrm{~K}$ for $5 \mathrm{Myr}$ according to the SIESS + COND isochrone) active accretors have smaller $\mathrm{Na} I E W \mathrm{~s}$ than non-accreting brown dwarfs. A first explanation of this trend would be that in the case of the accretting brown dwarfs our measurements of $\mathrm{Na} I E W \mathrm{~s}$ are affected by veiling; this, however, is not true because as described in Paper II, even for the sources with the most intense $\mathrm{H} \alpha$ emission, the veiling

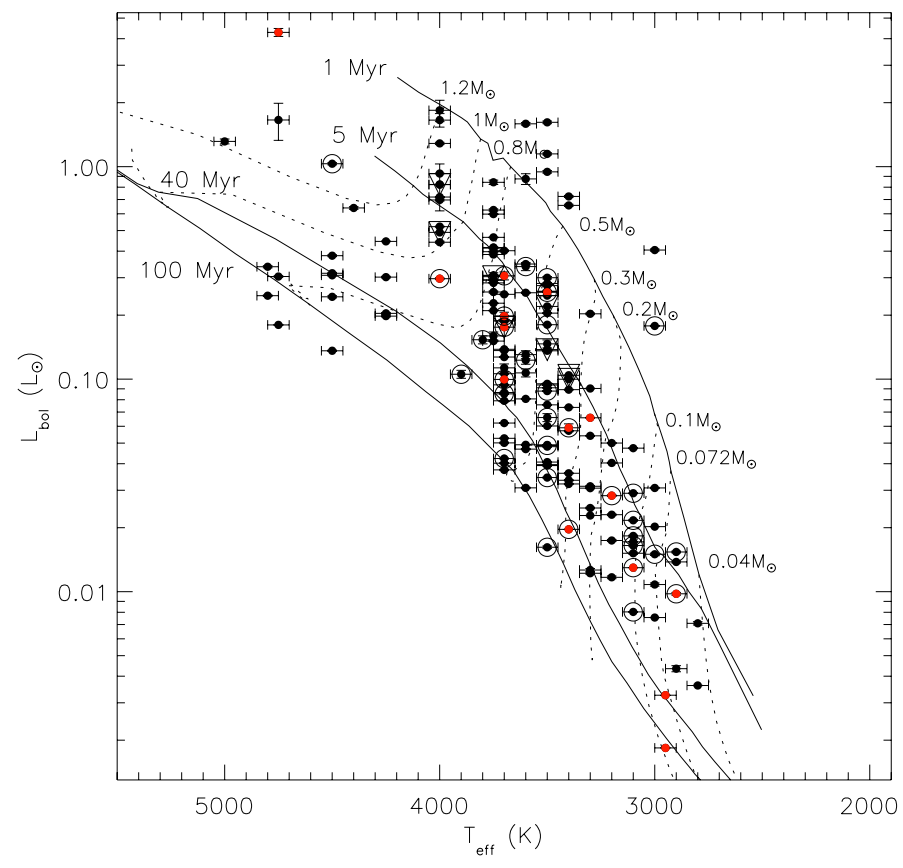

Fig. 11. HR diagram for the $\sim 200$ spectroscopically confirmed members by Dolan \& Mathieu (1999, 2001); Maxted et al. (2008); Sacco et al. (2008) or this work. We have overplotted several isochrones and evolutionary tracks from Baraffe et al. (1998). Symbols as in Fig. 10.

is minimal for wavelengths redder than $7400 \AA$. In any case, the sample is too small and the trend too shallow to allow us to perform any further analysis. Finally, the position of LOri115 in the previously mentioned diagram seems suspicious (it is the class II very low mass star that is clearly out of the general trend of the confirmed members), but its membership is discussed in the following section.

\subsection{Peculiar sources}

We distinguish in this section among four different groups: sources for which we have information about the Li absorption; objects for which we have information about other alkali lines (and/or emission lines and infrared excess); targets for which, owing to the lack of high resolution spectra and its "hot" nature, we had to use different criteria than alkali lines to study membership; and, finally, the coldest sample of sources only observed with SUBARU. 
A. Bayo et al.: Spectroscopy of very low mass stars and brown dwarfs in the Lambda Orionis star forming region

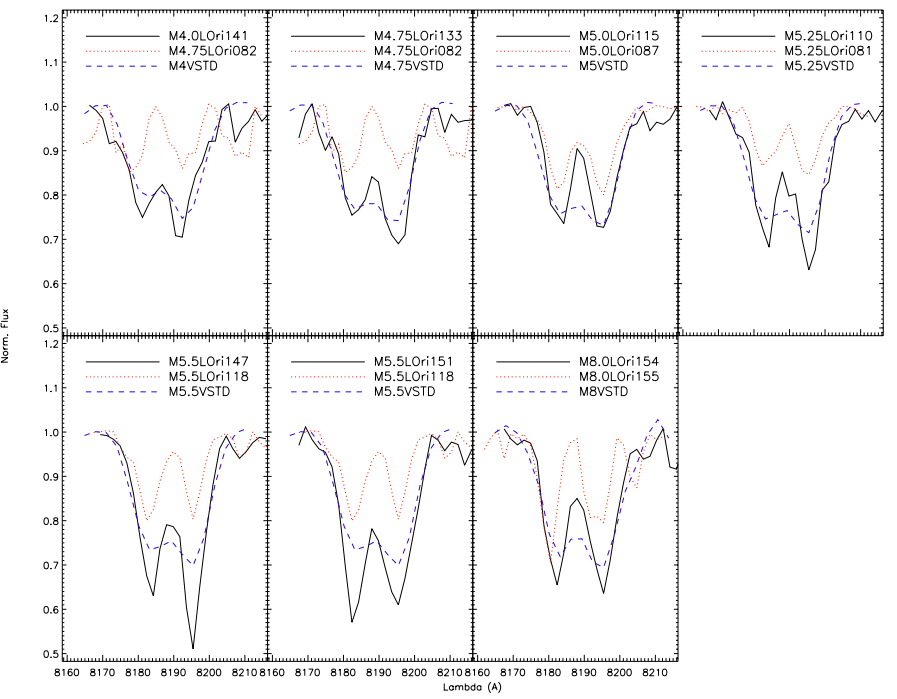

Fig. 12. Na I $\lambda 8200$ doublet detail for some of the particular sources (all but LOri115 discarded as members) with CAFOS spectra. For each case, we have overplotted in red a confirmed member with the same spectral type, and in blue a field dwarf (again with the same spectral type and obtained in the same campaign). Note that for spectral types M4.75 and M5.25, we have averaged spectra of field dwarfs with spectral types M4.5 and M5.0, M5.0 and M5.5, respectively.

\subsubsection{Sources with Li information}

LOri044, LOri046, LOri049, and LOri052 are all sources (except LOri052) that have been classified as non-members based on the absence or very marginal detection of $\mathrm{Li}$ I. In the cases of LOri044, LOri046, LOri049, and LOri052, either Maxted et al. (2008) or Sacco et al. (2008) also classify them as nonmembers based on their radial velocities. In our FLAMES spectra of LOri052, a marginal detection of Li I in absorption with $E W$ of $\sim 0.28 \AA$ was measured; this value is too low compared to other members of similar spectral type (even taking into account the general spread already commented on). Hence, in addition to the radial velocity determination of Maxted et al. (2008) and our alkali line study, we classify this source as a probable non-member.

For LOri007, no significant $\mathrm{H} \alpha$ emission was measured in the spectra of this source (observed with both TWIN and FLAMES), and although the equivalent width values of the $\mathrm{Na}$ I doublet is consistent with membership (see Table 6), no Li I in absorption was detected (see Table 6). Therefore we classify this source as non-member.

The sources DM065, DM070, DM061, LOri001, DM062, DM016, LOri026 (DM012), and LOri038 (DM002): have spectral types from K9 to M3, and were confirmed as members by Dolan \& Mathieu (1999) via Li absorption. In addition, their positions in the HR diagram are compatible with membership and most of them are X-ray emitters.

The source LOri1 15 displays a deeper Na I absorption (LRIS spectrum) than expected for its spectral type (M5) but a Li $E W$ (FLAMES spectrum) is perfectly compatible with membership. In addition, the SED of LOri115, exhibits a clear infrared excess at IRAC wavelength suggesting that the source is surrounded by an optically thick disk. It also shows quite strong and variable $\mathrm{H} \alpha$ emission ( $E W$ of -12.5 and -9 in our FLAMES and LRIS spectra), very close to the limit where pure activity cannot explain this emission. All these clear signs of youth allow us to classify it as a member and propose as a possible explanation of the deep $\mathrm{Na}$ I absorption some phenomenon related with activity.

\subsubsection{Other alkali lines and extra information}

The source LOri036 displays faint $\mathrm{H} \alpha$ emission, but the strengths of the alkali lines suggest that this object is older than the cluster members. Furthermore, Sacco et al. (2008) found a $\mathrm{RV}$ value that is not fully compatible with membership. For these two reasons, we classify this object as a possible non-member.

Neither of the sources LOri046 and LOri049 displays $\mathrm{H} \alpha$ emission. Both have very low gravity according to the alkali absorption strengths, and both had indeed been previously classified as possible non-members by Sacco et al. (2008) because of their measured radial velocity. We classify these sources no members.

The sources LOri110, LOri133, LOri141, LOri147, LOri151, LOri154 and LOri165 all have surface gravities (based on their measured $E W(\mathrm{NaI})$, see Table 6) that are far larger than those of cluster members. For the particular case of LOri110, no Li I was detected in our LRIS spectra, and Maxted et al. (2008) also measured $\mathrm{Na}$ I $E W$ s that were inconsistent with membership and confirmed that the derives radial velocities ruled out membership too. Barrado y Navascués et al. (2004b) classified LOri133, LOri151 and LOri165 as doubtful candidate members based on their photometric properties.

\subsubsection{Sources with spectral type earlier than M3 and no lithium data}

As verified by Slesnick et al. (2006), the Na I $8200 \AA$ doublet strength saturates for spectral types earlier than M2. Furthermore, the differences among dwarfs, giants, and young sources are very subtle even for M3 spectral types. Therefore, for these "hotter" sources where we did not have spectra of a resolution high enough to measure $\mathrm{Li}$, we used different sets of criteria to assess membership.

The non-members include C69-IRAC-010 and C69-IRAC008 that have spectral types F9 and $\mathrm{K} 3, \mathrm{H} \alpha$ in absorption, no infrared excess at the IRAC wavelength range, and a position in the HR diagram well below the $100 \mathrm{Myr}$ isochrone, hence are discarded them as members.

The following sources are classified as members:

- C69XE-009 (K2 spectral type) that displays a very intense $\mathrm{H} \alpha$ emission and an estimated position in the HR diagram that is perfectly compatible with membership;

- C69-IRAC-001 and C69-IRAC-002 (M1.5 and M3 spectral types, respectively): both are class II sources with thick disks according to the IRAC photometry and their positions in the HR diagram are compatible with membership;

- LOri024, LOri061, LOri048, and LOri062 (spectral types from M1.5 to M3): all of them were confirmed to be members by Sacco et al. (2008) and/or Maxted et al. (2008) based on their radial velocity (and in all the cases, their positions in the HR diagram are compatible with membership);

- C69-IRAC-007 and C69-IRAC-005 (M2.5 and M3): both sources are active accretors (see Paper II) with thick disks and positions in the HR diagram compatible with membership;

- C69XE-072 (M3 spectral type) which is detected in our X-ray survey (Barrado et al. 2011) and with an estimated position in the HR diagram compatible with membership. 

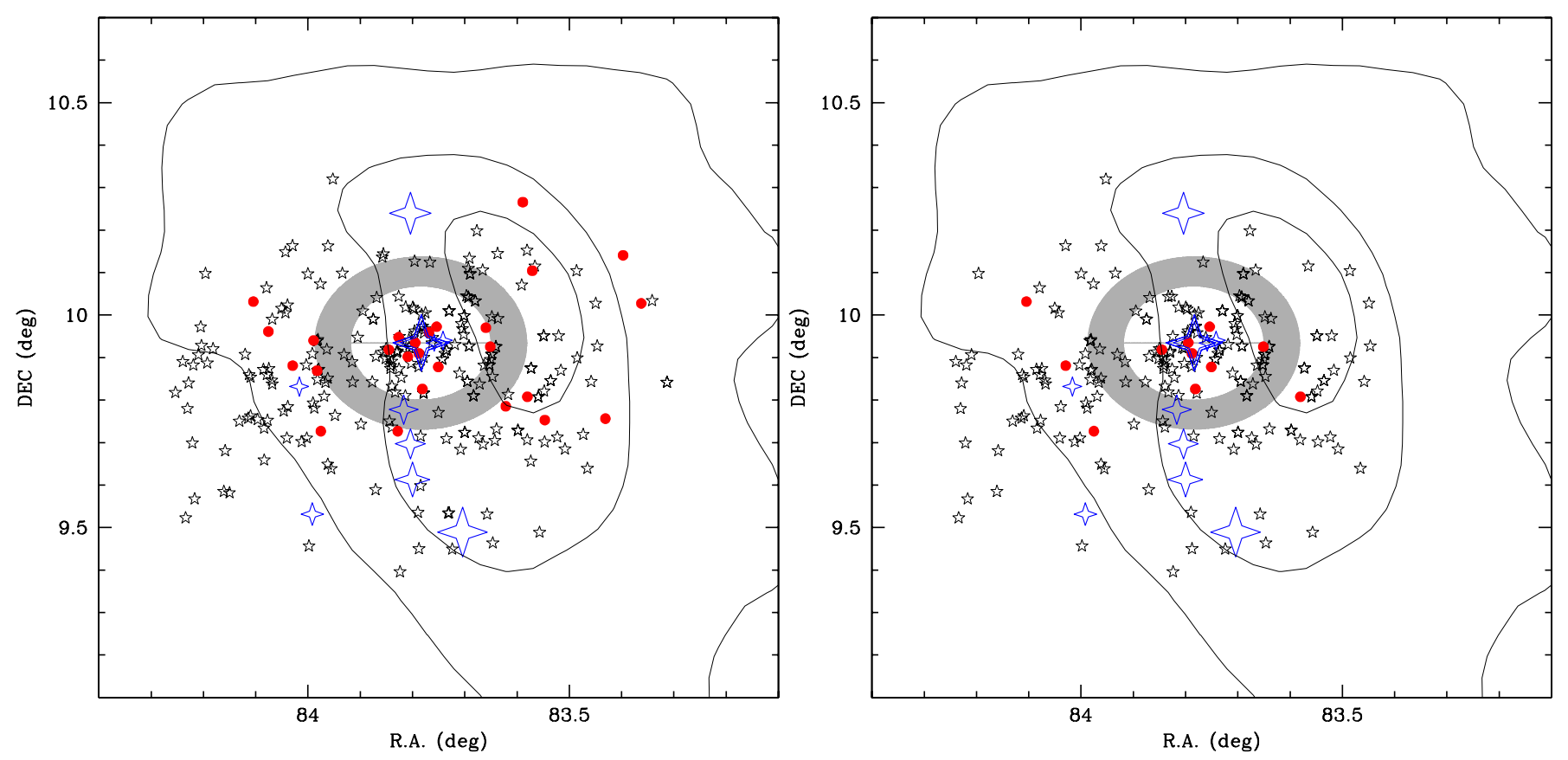

Fig. 13. Spatial distribution of in the left panel good photometric candidates and right panel spectroscopically confirmed members. The O and $\mathrm{B}$ populations are highlighted with large blue (four pointed) stars, members with masses above the $\mathrm{H}$ burning limit are displayed as small black (five pointed) stars, and brown dwarfs are indicated as filled red dots. In both figures, it is clear that brown dwarfs are not located at the edges of the cluster (as the ejection mechanism would predict). We have shaded in grey the ring corresponding to the void in the radial profile displayed on Fig. 14.

\subsubsection{Subaru L type sources}

The resolution of our Subaru/IRCS spectra was too low to resolve any alkali line. However, given the confirmed spectral types, the luminosities of our sources are not compatible with objects being much closer or much further than Lambda Orionis and therefore we assign a Y? membership only expecting to confirm definitely membership by means of, for example, a proper motion study on a longer time baseline than the one provided by the images from which these candidates were selected (we only have one set of Subaru images; see Morales-Calderón 2008)

To summarize and conclude the section, in Table 6 we have included a column with the final membership assigned to each source. We consider as members those labeled Y?: that is objects showing some peculiarity as already discussed but still considered as very good candidates for membership and we assign a label Y to spectroscopically confirmed members. In the latter case, we have included the reference of the work where the spectroscopic confirmation was achieved.

There are $\sim 240$ members from which 225 have been labeled as $\mathrm{Y}$ ? or Y (36 of them are confirmed as members of Collinder 69 for the first time in this work). These confirmed members are displayed in an HR diagram in Fig. 11. We note that there is a large dispersion in the diagram for the hotter part of the sample ( $T_{\text {eff }}$ higher than $\sim 3900 \mathrm{~K}$ ), where almost $100 \%$ of the candidates were confirmed by Dolan \& Mathieu $(1999,2001)$. However, if we take a closer look at the M (and cooler) population of Collinder 69 (on which this work is focused), the best-fit isochrone is that of $5 \mathrm{Myr}$ with an upper limit value of $20 \mathrm{Myr}$, which leads to the recovery of $95 \%$ of the confirmed members. The dispersion in this narrower area of the diagram, although smaller than that affecting hotter sources, is mainly explained by objects harboring disks and has been previously addressed in Bayo et al. (2008).
Focusing only on the spectroscopically confirmed members (from this work and the literature), we find a brown dwarf to star ratio $\left(R_{\mathrm{SS}}\right.$ as defined by Briceño et al. 2002) of 0.06 . This value is similar to that found for Taurus by Briceño et al. (2002) but significantly smaller than the revised one by Guieu et al. (2006) or the value reported for the ONC (Kroupa \& Bouvier 2003). This indicates that there are either environmental effects in the substellar formation efficiency (as suggested previously in, for example Briceño et al. 2002; Kroupa \& Bouvier 2003) or limitations in the spatial extension of our surveys.

\section{Mass segregation: ejection mechanism}

As mentioned in Sect. 1, one of the main goals of our long-term project on the LOSFR is to investigate which of the currently proposed mechanisms for the formation of brown dwarfs most closely agrees with the observations.

According to the ejection scenario of Reipurth \& Clarke (2001), brown dwarfs of an association such as Collinder 69 should be located mostly around the exterior parts of the cluster. If we assume an escape velocity of $\sim 1 \mathrm{~km} \mathrm{~s}^{-1}$ (as in the case of Taurus, Kroupa \& Bouvier 2003), after $\sim 5 \mathrm{Myr}$, the ejected brown dwarfs should still have been recovered by some of our photometric surveys (a $\sim 35^{\prime}$ distance is covered well by our SUBARU field and marginally by the Spitzer/IRAC one).

To test this hypothesis, we studied the spatial distribution of both stars and brown dwarfs in Collinder 69. In Fig. 13, we plot two diagrams corresponding to the distribution of stellar and substellar confirmed and candidate members to Collinder 69 (left panel for the whole set of confirmed members and good photometric candidates, and the right one only showing the spectroscopically confirmed members). In both panels, stellar sources are displayed as small black stars, whilst substellar objects are highlighted with red dots. Even though what we can see in this 


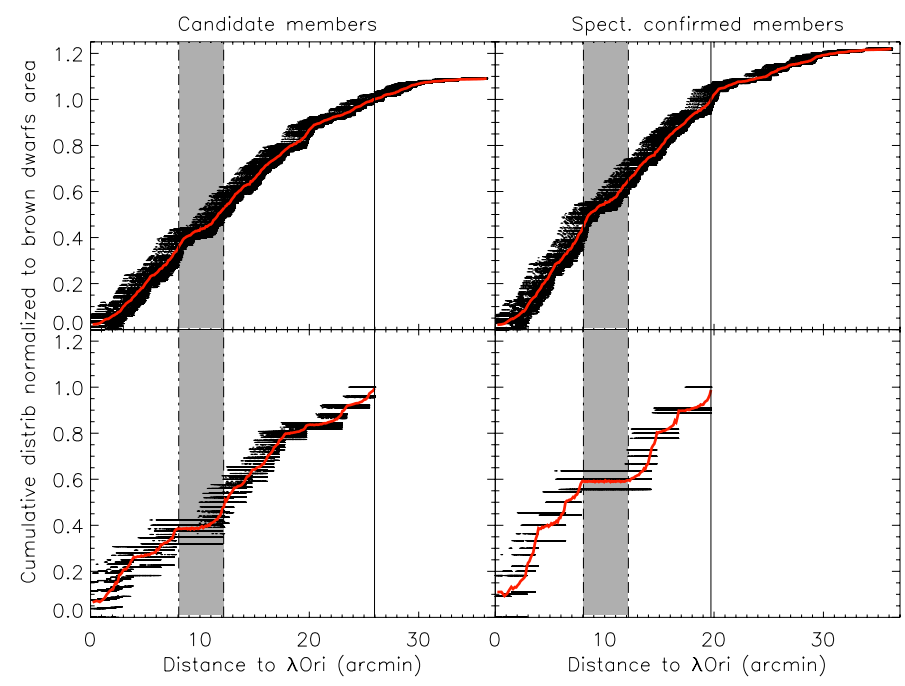

Fig. 14. Radial distribution of stars and brown dwarfs in Collinder 69: distance to $\lambda$ Ori vs. cumulative distribution of sources normalized to the radius where no more brown dwarfs are found in Collinder 69. As in the previous figure, we include two sets of panels: on the left side, candidates and confirmed members (top panel for stars and bottom for substellar objects) and on the right panel, only spectroscopically confirmed members (as in the previous case, top for stars and bottom for substellar sources). Note the obvious void (grey shaded area) of brown dwarfs (and not so obvious but still present for stars too) from $\sim 8-12$ arcmin distance to $\lambda$ Ori.

figure is nothing but a projection, any structured grouping of the substellar population is far from obvious.

From a more quantitative point of view, we tried to compare the distribution of stars and brown dwarfs with a twodimensional KS test, but because of the low numbers we are dealing with, the results were inconclusive. Trying a more simplistic approach, since we are mainly interested in the radial distribution of brown dwarfs, we compiled the cumulative radial distribution of stellar and substellar sources (good candidate members and spectroscopically confirmed members, as in Fig. 13).

Although simple, this analysis had to be done carefully taking into account again the low numbers we are dealing with. To obtain the cumulative distributions in a robust way, we performed $\sim 60$ different histograms using both multi-binning and multi-starting-point approaches and then smoothed the resulting function to ensure that the features found are bin and starting point independent.

We show the results of this method in Fig. 14, where the data-points of the individual histograms are drawn as black dots, and the smoothed final function is highlighted in red. It seems quite clear that there is no obvious differences between the distribution of stellar members of Collinder 69 (supposedly formed according to the classical paradigm) and the substellar ones. Furthermore, if we compare the distribution of brown dwarfs with that of objects that are clearly stars (masses higher than $0.5 M_{\odot}$ ), the results remain the same.

The only evident feature in these radial distributions is a void of both stars and brown dwarfs at $\sim 8-12$ arcmin distance from $\lambda$ Ori (projected $\sim 1-1.4 \mathrm{pc}$ ). We have highlighted this void in Fig. 13 where we see no correlation of this area with differences in dust densities as traced by the IRAS contours (the solid black lines).

To try to quantify the physical relevance of this void, we produced 100 synthetic two-dimensional (2D) normal populations of sources. For each population, we generated three different sets of data: stars, brown dwarfs, and star + brown dwarfs. To draw each set, we used the same number of objects that we have spectroscopically confirmed for each category in Collinder 69. The area from which the objects are drawn is the same area covered by our photometric surveys.

We run a one-dimensional KS test to compare the observed radial distribution with the synthetic ones (given the small number of brown dwarfs we analyzed exclusively the "only stars" and "stars + brown dwarfs" sets) and the result is that the probability of the observed radial distribution being drawn from a normal 2D population is negligible. Furthermore, only $7 \%$ of the synthetic radial distributions show voids similar in width (but closer to the edges of the cluster) to the one present in our observed distribution. Therefore, we are confident that this void is not merely a statistical artifact.

It is interesting and in disagreement with the ejection scenario that Collinder 69 seems more extended in the stellar population than in the substellar one (the vertical solid line in Fig. 14 marks the distance after which no more substellar members are found). This would indicate that the cluster has started to become dynamically relaxed, so low-mass members are falling into the gravitational well. An alternative explanation, given the very young age of the cluster, would be that the brown dwarfs might just have formed preferably closer to the center. As a note regarding the possible effect of the spatial coverage of our surveys, we wish to point out that even though our SUBARU field extends further away from Lambda Orionis than the other surveys, we do not find brown dwarf candidates more distant from this association than $\sim 20$ arcmin.

In any case, we recall once more that we are are affected by the small numbers of objects and that our photometric surveys are not wide enough to recover the possible ejected brown dwarfs if their escape velocities are twice those estimated for Taurus. Therefore, the most we can conclude from our analysis is that the ejection mechanism of formation does not seem to be able to explain the observed properties of Collinder 69 for escape velocities of $\sim 1 \mathrm{~km} \mathrm{~s}^{-1}$ or smaller.

\section{The initial mass function for spectroscopically confirmed members}

To derive the mass of the members of Collinder 69, we used the estimated $T_{\text {eff }}$ and $L_{\text {bol }}$ from the SED fit along with a composite 5 Myr isochrone (Siess et al. $2000+$ COND from Baraffe et al. 2003). The average mass resulting from these two estimates for each object is provided in Table 6. As can be seen in Fig. 15, there is a quite large dispersion when comparing the estimates. If we separate our members according to the ratio of the two estimated masses into "large dispersion" (those where one of the masses is higher than 1.5 times the other) and "acceptable" dispersion (the ratio of the highest to the lowest mass lower than 1.5); we find that $\sim 30 \%$ of the so-called "large dispersion" sources have been classified as class II according to their IRAC colours, while the same class in the acceptable dispersion sample decreases to $15 \%$ (this is not a question of scale since both samples have a similar number of sources). Indeed, $\sim 65 \%$ of the sources undergoing active accretion fall into the large dispersion sample. Therefore, our mass estimates are quite sensitive to the presence of disks (a caveat that does not surprise us, as discussed in Bayo et al. 2008). To take into account this uncertainty, we present all the calculations for both estimates of the mass of the members and for the average masses. 


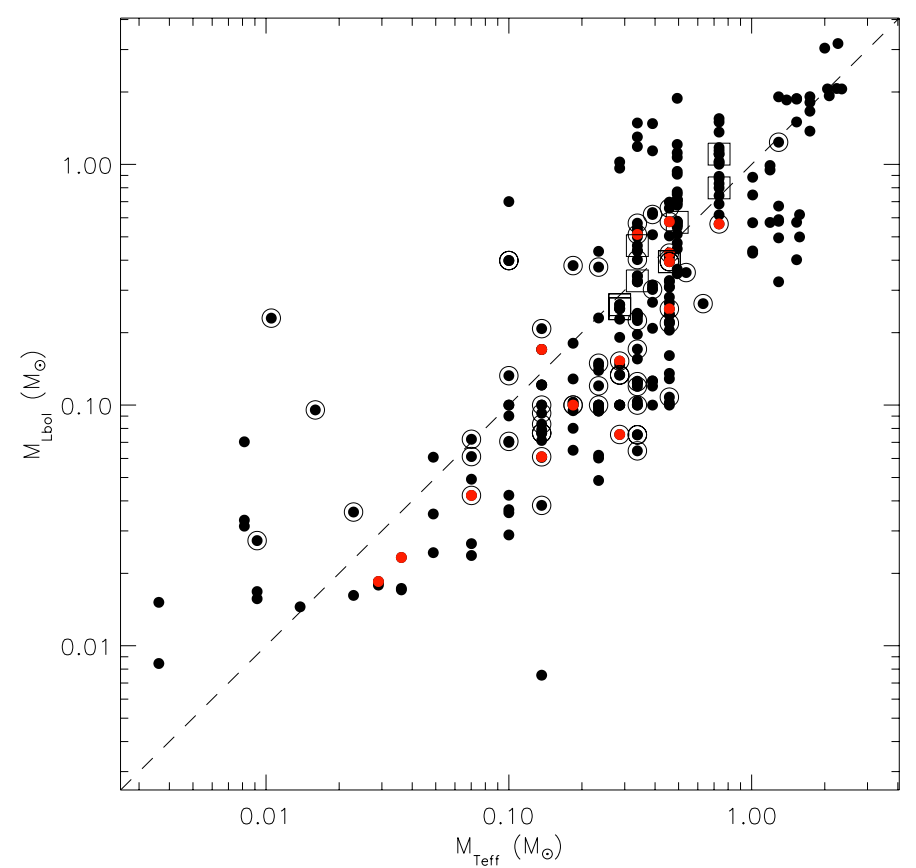

Fig. 15. Relationship between the masses derived from $T_{\text {eff }}$ and $L_{\text {bol }}$. Members (spectroscopically confirmed) and candidates (photometric) are displayed as solid dots. As in previous figures, we have highlighted peculiar sources (class II, binaries and accretors).

Table 5. Fitted slopes for the IMF of Collinder 69 taking into account the different methods to estimate the mass of the confirmed members (see text for further details).

\begin{tabular}{lcc}
\hline \hline $\begin{array}{l}\text { Estimated } \alpha \text { for } \\
\text { the mass range: }\end{array}$ & $25-0.65 M_{\odot}$ & $0.65-0.01 M_{\odot}$ \\
\hline C69 IMF (I) & 1.73 & 0.18 \\
C69 IMF (II) & 1.81 & 0.38 \\
C69 IMF (III) & 1.88 & 0.31 \\
C69 IMF (IV) & 1.79 & 0.16 \\
C69 IMF (V) & 1.85 & 0.38 \\
C69 IMF (VI) & 1.85 & 0.33 \\
\hline SOri ${ }^{1}$ & 0.8 \\
APer & \multicolumn{2}{c}{0.6} \\
M 35 $^{2,3}$ & \multicolumn{3}{c}{$(0.81)-(-0.88)$} \\
Pleiades & \multicolumn{2}{c}{0.6} \\
\hline
\end{tabular}

Notes. We also include the slopes estimated for low mass stars (the mass ranges are not always the same ones but roughly $M_{*}<0.8 M_{\odot}$ ) in other studies of different open clusters. Slope estimated with data from: ${ }^{(1)}$ Béjar et al. (2001); ${ }^{(2)}$ Barrado y Navascués et al. (2001a); (3) Barrado y Navascués et al. (2002); (4) Barrado y Navascués et al. (2001b); ${ }^{(5)}$ Bouvier et al. (1998).

In the left panels of Fig. 16, we present the derived initial mass functions (according to the different procedures followed to derive the masses of the sources) for Collinder 69. For this first case, we considered only spectroscopically confirmed members confined within the intersection of the CFHT, Spitzer, and XMM fields of view (see Fig. 1). For the assembly of each IMF, in the range of masses covered by this work, we proceeded in a similar manner as in the previous section with the multi-binning, multi-starting-point approach. In addition, to enlarge the mass range coverage, we included data from Murdin \& Penston (1977) (scaling to the respective areas) as open blue circles. The last point at the massive end corresponds

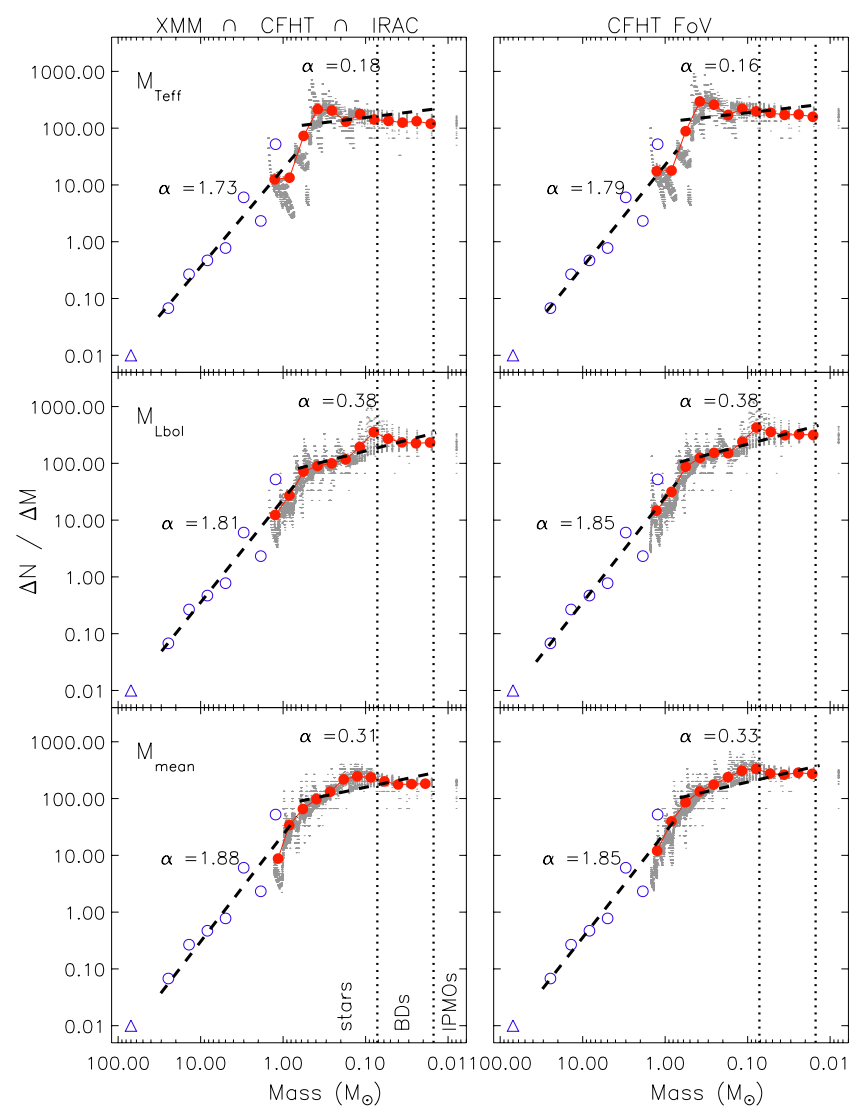

Fig. 16. IMFs derived for Collinder 69 taking into account three different determination of mass for each member and the two different areas within the cluster. Left panels: area confined within the intersection of the XMM, CFHT, and IRAC fields of view. Top: IMF (I). Masses derived combining the estimated $T_{\text {eff }}$ and the composite $5 \mathrm{Myr}$ isochrone (SIESS + COND). Middle: IMF (II). Masses derived combining the estimated $L_{\mathrm{bol}}$ and the composite $5 \mathrm{Myr}$ isochrone (SIESS + COND). Bottom: IMF (III). Masses derived averaging the masses from the previous two figures. Right panels: area confined within the CFHT field of view (including the central part of the cluster). Top: IMF (IV). Same method to estimate masses as in IMF (I). Middle: IMF(V). Same method to estimate masses as in IMF (II). Bottom: IMF (VI). Same method to estimate masses as in IMF (III).

to a possible SN (open blue triangle, see discussion in Dolan \& Mathieu 1999).

We note that the previously described area (the intersection of the three FoVs), does not contain the central part of the cluster (where the O8 III binary star, $\lambda$ Ori, is located). To study the possible effects of this omission, in the right panels of Fig. 16 we show another set of IMFs (again three for the different estimated masses), this time focused on the CFHT field of view (scaling the XMM members to the corresponding area).

Regardless of the parameter used to infer the mass of our confirmed and candidate members, for masses above $0.65 M_{\odot}$, the power law index of the IMF is similar to Salpeter's value, being much smaller for lower masses. In particular, the fitted slopes in the mass range $25-0.70 M_{\odot}$ vary from 1.73 to 1.89 , and those for the low and very low mass range, i.e., $0.70-0.01 M_{\odot}$, correspond to much lower values from 0.16 to 0.38 (see Table 5 for a summary of the slopes).

As we previously mentioned, the two areas for which we derived the IMFs (the CFHT survey coverage and the intersection of the CFHT, Spitzer and XMM fields of view) comprise populations that show distinct trends in terms of spatial distribution (the 


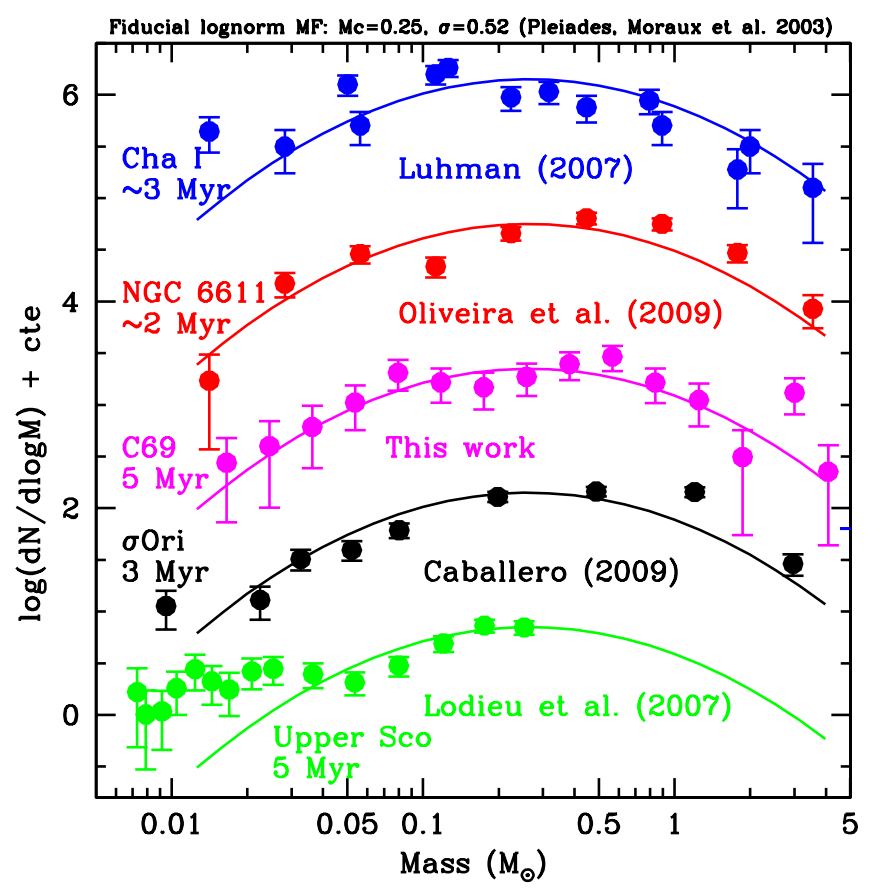

Fig. 17. Log-log representation of the IMF for different young associations. We compare our very complete spectroscopically confirmed IMF (in pink) with photometric ones derived for associations with similar ages ( 2-5 Myr, Luhman et al. 2007; Oliveira et al. 2009; Caballero et al. 2009; Lodieu et al. 2007). The compilation of the data corresponding to clusters other than Collinder 69 are courtesy of Bouvier and Moraux.

XMM coverage does not include a relatively large population of class II sources). Surprisingly, this does not seem to affect the shape of the IMF since the slopes shown in Fig. 16 appear to be consistent with each other.

Our estimated slopes are systematically lower (but in a similar range) than those derived for photometrically compiled IMFs for the open clusters SOri, APer, M35, and the Pleiades (see Table 5 for the exact numbers and references). For the low mass stars/substellar domain, the differences are not too large, regardless of the total mass, the environments, and the age of each association. The only exception to this is M 35 - which corresponds to the core of the cluster -, a very rich association, about 150 Myr old, where important dynamical evolution might have taken place.

In particular, if we only compare with similar age clusters, as we do in Fig. 17, we can see that our IMF (in this case we have used the mean mass determination for the intersection of the three fields of view) looks very similar to that of, for example, NGC 6611 with, in our case, a cut-off at $\sim 0.016 M_{\odot}$, where we think (because of the completeness of our photometric surveys) that we have reached the minimum mass for members of Collinder 69.

\section{Conclusions}

We have analyzed $\sim 170$ optical and near infrared spectra with a wide range of resolutions of candidate members to the Collinder 69 cluster. On the basis of different criteria regarding molecular absorption bands, we have provided spectral types for all sources. Using alkali lines as youth indicators, we have confirmed 90 members (and 9 possible members). For those sources of our survey overlapping with radial velocity surveys (by
Sacco et al. 2008; Maxted et al. 2008), we obtained very similar results regarding membership. A summary of the sources analyzed in this work can be found in Table 6 .

We have confirmed the cool nature of the lowest mass candidate members of Collinder 69 reported so far. With derived spectral types L0-L2 corresponding to effective temperatures of $\sim 2000 \mathrm{~K}$ (according to the spectroscopic temperature scales derived by Basri et al. 2000), if they are members, they have estimated masses, following the 5 Myr DUSTY isochrone by Chabrier et al. 2000, as low as $0.016 M_{\odot}\left(\sim 16.5 M_{\text {Jupiter }}\right)$.

We have compiled the most complete IMF based on spectroscopically confirmed members for a young association and when compared with others (photometric IMFs), no significant differences in the low mass and substellar domain have been identified.

With our study of the spatial distribution of confirmed stellar and substellar members of Collinder 69, we provided some caveats to the ejection formation scenario for brown dwarfs, in order for it to be feasible in this region.

Acknowledgements. We wish to thank the anonymous referee for very useful comments that improved the manuscript without doubt. This research has been funded by Spanish grants ESP2007-65475-C02-02, CSD2006-00070, PRICITS2009/ESP-1496 and AYA 2010-21161-C02-02. The support of the ESAC faculty is also recognized. It makes use of VOSA, developed under the Spanish Virtual Observatory project supported from the Spanish MICINN through grant AyA2008-02156, and of the SIMBAD database, operated at CDS, Strasbourg, France. The authors wish to thank J. Bouvier and E. Moraux for their data compilation on IMFs of different young associations and Y. Momany for the interesting discussion about statistics when building histograms. This research has made use of the Keck Observatory Archive (KOA), which is operated by the W. M. Keck Observatory and the NASA Exoplanet Science Institute (NExScI), under contract with the National Aeronautics and Space Administration. This paper includes data gathered with the $6.5 \mathrm{~m}$ Magellan Telescopes located at Las Campanas Observatory, Chile. Based on observations made with ESO Telescopes at the La Silla Paranal Observatory under programmes ID 080.C-0592 and 078.C-0124. Based on observations collected at the Centro Astronómico Hispano Alemán (CAHA) at Calar Alto, operated jointly by the Max-Planck Institut für Astronomie and the Instituto de Astrofísica de Andalucía (CSIC). Based in part on data collected at Subaru Telescope, which is operated by the National Astronomical Observatory of Japan.

\section{References}

Allard, F., Hauschildt, P. H., Alexander, D. R., Tamanai, A., \& Schweitzer, A. 2001, ApJ, 556, 357

Allard, F., Guillot, T., Ludwig, H.-G., et al. 2003, in IAU Symp. 211, Brown Dwarfs, ed. E. Martín, 325

Baraffe, I., \& Chabrier, G. 2010, A\&A, 521, A44

Baraffe, I., Chabrier, G., Allard, F., \& Hauschildt, P. 1998, VizieR Online Data Catalog, 333, 70403

Baraffe, I., Chabrier, G., Allard, F., \& Hauschildt, P. H. 2002, A\&A, 382, 563

Baraffe, I., Chabrier, G., Barman, T. S., Allard, F., \& Hauschildt, P. H. 2003, A\&A, 402, 701

Barrado y Navascués, D., \& Martín, E. L. 2003, AJ, 126, 2997

Barrado y Navascués, D., Stauffer, J. R., \& Patten, B. M. 1999, ApJ, 522, L53

Barrado y Navascués, D., García López, R. J., Severino, G., \& Gomez, M. T. 2001a, A\&A, 371, 652

Barrado y Navascués, D., Stauffer, J. R., Bouvier, J., \& Martín, E. L. 2001b, ApJ, 546, 1006

Barrado y Navascués, D., Zapatero Osorio, M. R., Martín, E. L., et al. 2002, A\&A, 393, L85

Barrado y Navascués, D., Mohanty, S., \& Jayawardhana, R. 2004a, ApJ, 604, 284

Barrado y Navascués, D., Stauffer, J. R., Bouvier, J., Jayawardhana, R., \& Cuillandre, J.-C. 2004b, ApJ, 610, 1064

Barrado y Navascués, D., Stauffer, J. R., Morales-Calderón, M., et al. 2007, ApJ, 664,481

Barrado, D., Stelzer, B., Morales-Calderón, M., et al. 2011, A\&A, 526, A21

Basri, G. 1997, Mem. Soc. Astron. Ital., 68, 917

Basri, G., Mohanty, S., Allard, F., et al. 2000, ApJ, 538, 363

Bayo, A. 2009, Ph.D. Thesis, Universidad autónoma de madrid 
Bayo, A., Rodrigo, C., Barrado Y Navascués, D., et al. 2008, A\&A, 492, 277 Béjar, V. J. S., Martín, E. L., Zapatero Osorio, M. R., et al. 2001, ApJ, 556, 830 Bessell, M. S., Castelli, F., \& Plez, B. 1998, A\&A, 337, 321

Bildsten, L., Brown, E. F., Matzner, C. D., \& Ushomirsky, G. 1997, ApJ, 482, 442

Bouvier, J., Stauffer, J. R., Martin, E. L., et al. 1998, A\&A, 336, 490

Briceño, C., Luhman, K. L., Hartmann, L., Stauffer, J. R., \& Kirkpatrick, J. D. 2002, ApJ, 580, 317

Burrows, A., Marley, M., Hubbard, W. B., et al. 1997, ApJ, 491, 856

Caballero, J. A., López-Santiago, J., de Castro, E., \& Cornide, M. 2009, AJ, 137, 5012

Castelli, F., Gratton, R. G., \& Kurucz, R. L. 1997, A\&A, 318, 841

Chabrier, G., \& Baraffe, I. 1997, A\&A, 327, 1039

Chabrier, G., Baraffe, I., Allard, F., \& Hauschildt, P. 2000, ApJ, 542, 464

Comerón, F., Neuhäuser, R., \& Kaas, A. A. 2000, A\&A, 359, 269

Cruz, K. L., \& Reid, I. N. 2002, AJ, 123, 2828

da Silva, L., Torres, C. A. O., de La Reza, R., et al. 2009, A\&A, 508, 833

Dolan, C. J., \& Mathieu, R. D. 1999, AJ, 118, 2409

Dolan, C. J., \& Mathieu, R. D. 2001, AJ, 121, 2124

Dolan, C. J., \& Mathieu, R. D. 2002, AJ, 123, 387

Duerr, R., Imhoff, C. L., \& Lada, C. J. 1982, ApJ, 261, 135

Fernández, M., \& Comerón, F. 2001, A\&A, 380, 264

Geballe, T. R., Knapp, G. R., Leggett, S. K., et al. 2002, ApJ, 564, 466

Gomez, M., \& Lada, C. J. 1998, AJ, 115, 1524

Gómez, M., \& Persi, P. 2002, A\&A, 389, 494

Guieu, S., Dougados, C., Monin, J.-L., Magnier, E., \& Martín, E. L. 2006, A\&A, 446, 485

Hauschildt, P. H., Allard, F., \& Baron, E. 1999, ApJ, 512, 377

Jeffries, R. D., \& Oliveira, J. M. 2005, MNRAS, 358, 13

Jeffries, R. D., Oliveira, J. M., Barrado y Navascués, D., \& Stauffer, J. R. 2003, MNRAS, 343, 1271

Jeffries, R. D., Jackson, R. J., James, D. J., \& Cargile, P. A. 2009, MNRAS, 400, 317

Jones, H. R. A., Longmore, A. J., Jameson, R. F., \& Mountain, C. M. 1994, MNRAS, 267, 413

Kroupa, P., \& Bouvier, J. 2003, MNRAS, 346, 369
Leggett, S. K., Allard, F., Dahn, C., et al. 2000, ApJ, 535, 965

Lodieu, N., Hambly, N. C., Jameson, R. F., et al. 2007, MNRAS, 374, 372

Lodieu, N., Hambly, N. C., Jameson, R. F., \& Hodgkin, S. T. 2008, MNRAS, 383,1385

Luhman, K. L. 1999, ApJ, 525, 466

Luhman, K. L., Liebert, J., \& Rieke, G. H. 1997, ApJ, 489, L165

Luhman, K. L., Stauffer, J. R., Muench, A. A., et al. 2003, ApJ, 593, 1093 Luhman, K. L., Adame, L., D’Alessio, P., et al. 2007, ApJ, 666, 1219

Martin, E. L., Rebolo, R., \& Zapatero-Osorio, M. R. 1996, ApJ, 469, 706

Martín, E. L., Delfosse, X., Basri, G., et al. 1999, AJ, 118, 2466

Maxted, P. F. L., Jeffries, R. D., Oliveira, J. M., Naylor, T., \& Jackson, R. J. 2008, MNRAS, 385, 2210

Mohanty, S., Jayawardhana, R., \& Basri, G. 2005, ApJ, 626, 498

Morales-Calderón, M. 2008, Ph.D. Thesis, Universidad autónoma de madrid

Mouschovias, T. C. 1991, NATO ASIC, Proc. 342, 449

Murdin, P., \& Penston, M. V. 1977, MNRAS, 181, 657

Natta, A., Testi, L., Muzerolle, J., et al. 2004, A\&A, 424, 603

Neuhaeuser, R., Wolk, S. J., Torres, G., et al. 1998, A\&A, 334, 873

Oliveira, J. M., Jeffries, R. D., \& van Loon, J. T. 2009, MNRAS, 392, 1034

Padoan, P., \& Nordlund, A. 2002, ApJ, 576, 870

Reid, I. N., \& Gizis, J. E. 1997, AJ, 113, 2246

Reid, I. N., Hawley, S. L., \& Gizis, J. E. 1995, AJ, 110, 1838

Reipurth, B., \& Clarke, C. 2001, AJ, 122, 432

Sacco, G. G., Franciosini, E., Randich, S., \& Pallavicini, R. 2008, A\&A, 488, 167

Schiavon, R. P., Barbuy, B., Rossi, S. C. F., \& Milone, A. 1997, ApJ, 479, 902

Shu, F. H., Adams, F. C., \& Lizano, S. 1987, ARA\&A, 25, 23

Siess, L., Dufour, E., \& Forestini, M. 2000, A\&A, 358, 593

Slesnick, C. L., Carpenter, J. M., \& Hillenbrand, L. A. 2006, AJ, 131, 3016

Stauffer, J. R., Schultz, G., \& Kirkpatrick, J. D. 1998, ApJ, 499, L199

Stauffer, J. R., Barrado y Navascués, D., Bouvier, J., et al. 1999, ApJ, 527, 219

Vacca, W. D., Cushing, M. C., \& Rayner, J. T. 2003, PASP, 115, 389

Ventura, P., Zeppieri, A., Mazzitelli, I., \& D’Antona, F. 1998, A\&A, 331, 1011

Whitworth, A. P., \& Zinnecker, H. 2004, A\&A, 427, 299

Wilking, B. A., Greene, T. P., \& Meyer, M. R. 1999, AJ, 117, 469

Zapatero Osorio, M. R., Béjar, V. J. S., Pavlenko, Y., et al. 2002, A\&A, 384, 937 


\section{Appendix A: Automatic line characterization procedure and resolution effects}

In brief; one of the most difficult steps in automating the measurement of line properties is the determination of the continuum on top of (or below) which the line lies. Even when this task is performed manually, different astronomers might select different points to represent the continuum when, for example, analyzing a noisy spectrum or a line embedded in a molecular band. The code that we have developed tries to solve this problem by proceeding in an iterative and consistent way.

First of all, it locates where the actual peak of the line is. In this step, the wavelength calibration accuracy is taken into account providing limits to the wavelength coverage where the search for the peak of the line is carried out. Once this task is accomplished, two regions are defined (to the right and to the left of the estimated central wavelength) and the width of the regions is fixed to 100 points to be statistically significant: the width in wavelength units therefore depends on the resolution of the spectra. A linear fit using those regions is performed (red dotted line in Fig. A.1). With this attempt to define a continuum (the linear fit), the code calculates a first guess of the FWHM (red cross in Fig. A.1).

For the second iteration, the code considers that the line is a Gaussian, and, therefore that $F W H M=2 \times \sqrt{2 \times \log (2)} \times \sigma$. Assuming that at a distance of $\pm 10 \sigma$, one should be outside the line, two new regions in the spectra are selected, starting at $\pm 10 \sigma$ from the wavelength of the peak and ending at the same limits as before. These two regions are close to the edges of the line unless the line has a very wide double-peaked structure.

At this point, the code will perform two linear fits in a sequential way; a second continuum is derived with a linear fit to these new regions of the spectra close to the edges of the line, and the third one with another linear fit, but this time considering only those data points that differ from the second continuum by less than one dispersion of the difference between the actual spectra and this second continuum (the blue dots in Fig. A.1 represent the data points considered to define a third continuum and the blue dashed line is the resulting continuum fit).

Once the third guess of the locus of the continuum is estimated, the code calculates a second iteration of the FWHM (blue cross in Fig. A.1). Assuming one more time that the line can be described with a Gaussian (and calculating the associated $\sigma$ ), it considers three pairs of wavelengths in the spectra to make the final measurements (pairs located at $\pm 3 \sigma, \pm 4 \sigma$ and $\pm 5 \sigma$ from the wavelength of the peak). As a final refinement, for each selected pair of wavelengths, for example $\lambda_{1}$ and $\lambda_{2}$ corresponding to $\lambda_{\text {peak }} \pm 3 \sigma$, the code checks whether the linear continuum defined by $\left(\lambda_{1}, F\left(\lambda_{1}\right)\right),\left(\lambda_{2}, F\left(\lambda_{2}\right)\right)$ intersects the wings of the line; when this is the case (for example the upper-left panel of Fig. A.1), the code uses the third continuum to perform the measurements.

Prior to providing these measurements, the code subtracts the instrumental profile from the values estimated for the FWHM and $\mathrm{FW}_{10 \%}$ considering that the FWHM of the convolution $\left(G_{\text {measured }}\right)$ of two Gaussians $\left(G_{\text {instr }}, G_{\text {line }}\right)$ is given by:

$$
F W H M\left(G_{\text {measured }}\right)=\sqrt{F W H M\left(G_{\text {instr }}\right)^{2}+F W H M\left(G_{\text {line }}\right)^{2}}
$$

and that FWHM and $\mathrm{FW}_{10 \%}$ are related by: $\mathrm{FW}_{10 \%}=\sqrt{\frac{\log (2)}{\log (10)}} \times F W H M$.

The final product is the mean of the three measurements for each parameter $\left(F W H M, \mathrm{FW}_{10 \%}\right.$, and $\left.E W\right)$ and their corresponding standard deviation $\sigma$.
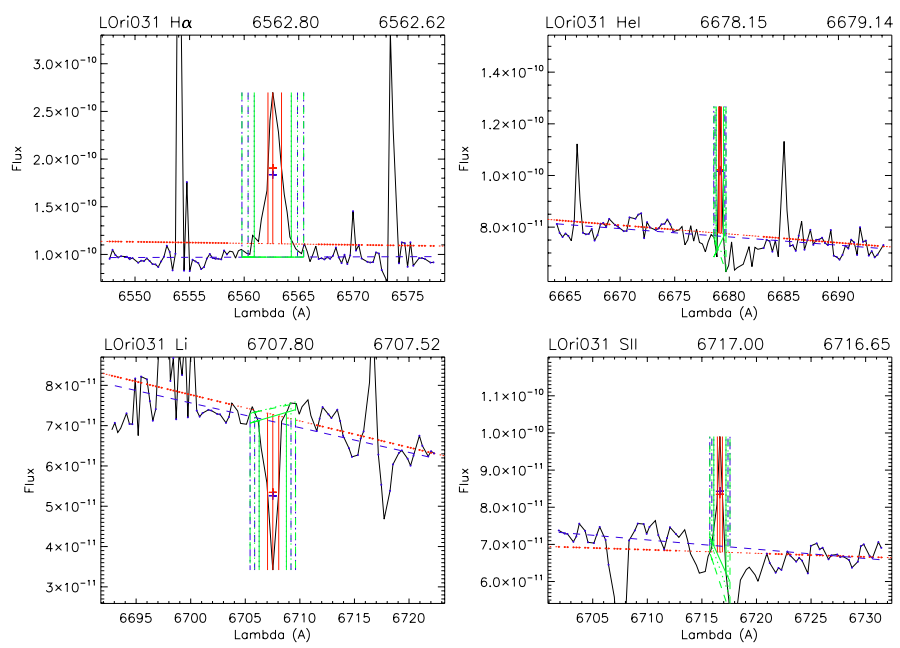

Fig. A.1. Example of the output of the code designed to measure $E W$, $F W H M$, and $\mathrm{FW}_{10 \%}$ of the different lines detected on the spectra in an automatic manner. In this case, we show four of the measured lines in the average MIKE spectra obtained for LOri031: $\mathrm{H} \alpha$ at $6562.80 \AA$, He I at $6678.15 \AA$, Li I at $6707.8 \AA$, and S II at $6717.0 \AA$. For each case, the canonical wavelength for the measured line and the actual wavelength where the line has been found in our spectra are displayed as the title of the plot (all of them within the errors in the wavelength calibration). The iterative process in the continuum determination is displayed using different colors: red for the first iteration (including the first guess for the half maximum flux value, red cross), blue for the second one (filled blue dots on the data-points used to refine the first continuum, blue cross showing the second half maximum estimation), and the three green lines (filled, dotted and dashed) representing the final determined continuum.

Once we had an automatic procedure to characterize the lines present in our spectra, we had to address the effect that the wide dispersion in resolution might introduce to our measurements. We proceeded in the following manner: we degraded one of our FLAMES spectra (from LOri038, a M3, accreting, Class II, confirmed member) to different resolutions (including those listed in Table 1). We then used (on those degraded spectra) our code to measure the $F W H M, E W$, and $\mathrm{FW}_{10 \%}$ of the $\mathrm{H} \alpha$ (emission) and Li $26708 \AA$ lines (absorption). In Fig. A.2, we provide these values $\left(E W\right.$ and $\mathrm{FW}_{10 \%}$ for $\mathrm{H} \alpha$ and $E W$ for $\left.\mathrm{Li} \mathrm{I} \lambda 6708 \AA\right)$ as a function of the resolution of the spectra. The length of the $y$ axes has been intentionally fixed for each plot: in the first panel (from left to right), it represents the variation in the instrumental response (the FWHM measured on the respective arc adapted for each value of the resolution) among the resolutions considered. In the cases of the middle and right-side panels, the length of these axes provides an idea of the variation within members of Collinder 69 for the corresponding equivalent width. We note that in the case of $\mathrm{H} \alpha$ there is clearly no significant dependence on the measurements made at different resolutions (the variations lie within the error bars). For the case of lithium, owing to the intrinsic weakness of the line, the accuracy of the measurement (meaning the error bars) lowers with the spectral resolution, but it still seems perfectly reasonable to compare measurements at $R \sim 2000$ and $R \sim 8000$. In the same figure, we have highlighted the range of resolutions where $\mathrm{Li}$ is no longer detectable. A detailed view of the "degeneration" of the lines for this example is provided in Fig. A.3 

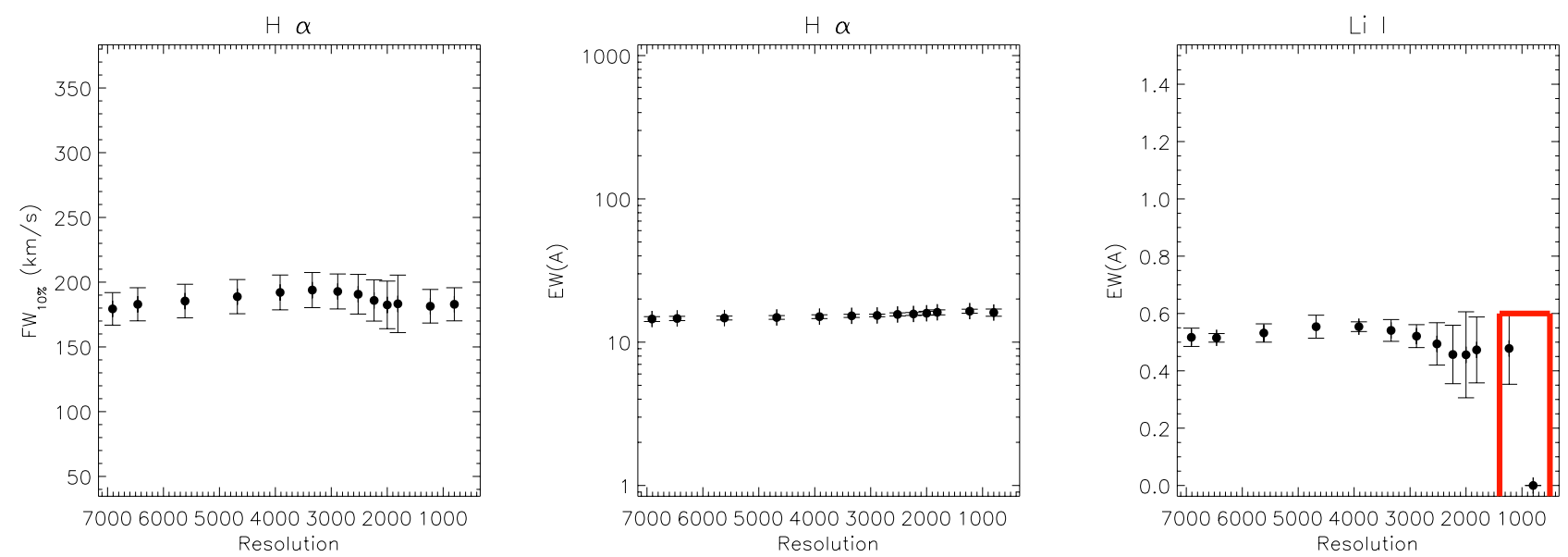

Fig. A.2. Relationship between different measurements of the $\mathrm{H} \alpha$ and Li I line profiles and the resolution of the spectra where the measurement has been performed.

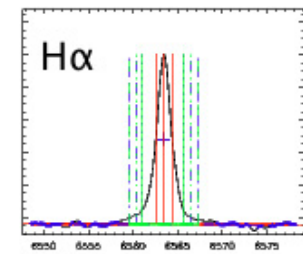

$\mathrm{R} \sim 8000$

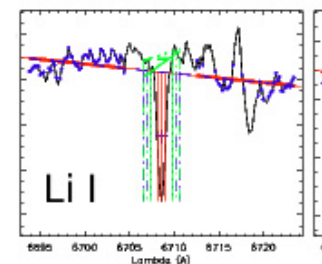

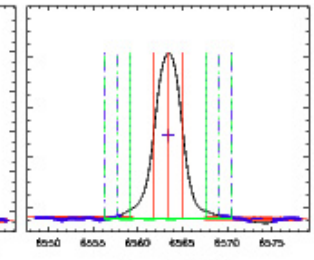
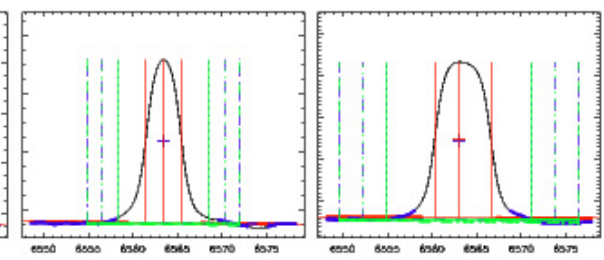

$R \sim 1250$

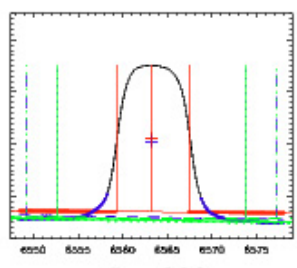

$\mathrm{R} \sim 2000$
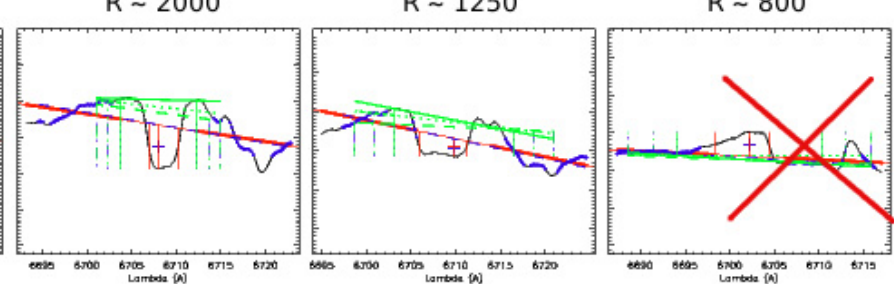

Fig. A.3. Sequence providing a detailed view of the evolution of the profiles of the H $\alpha$ (emission) and Li I (absorption) lines with the variation in the spectral resolution. The first panel (from left to right) corresponds to the original resolution of our FLAMES spectrum of LOri038, a M3 member of C69 $(R \sim 8000)$. In the last panel, we have degraded the FLAMES spectra to a resolution similar to that of our low-resolution campaign ( $R \sim 800$ obtained with the B\&C spectrograph). As can be seen in this figure and in Fig. A.2 the lithium equivalent width can be measured down to resolutions of the order of 1250 (for a high $\mathrm{S} / \mathrm{N}$ as in the case of our spectrum of LOri038). The linestyles and color code are those explained in the text. 
A. Bayo et al.: Spectroscopy of very low mass stars and brown dwarfs in the Lambda Orionis star forming region

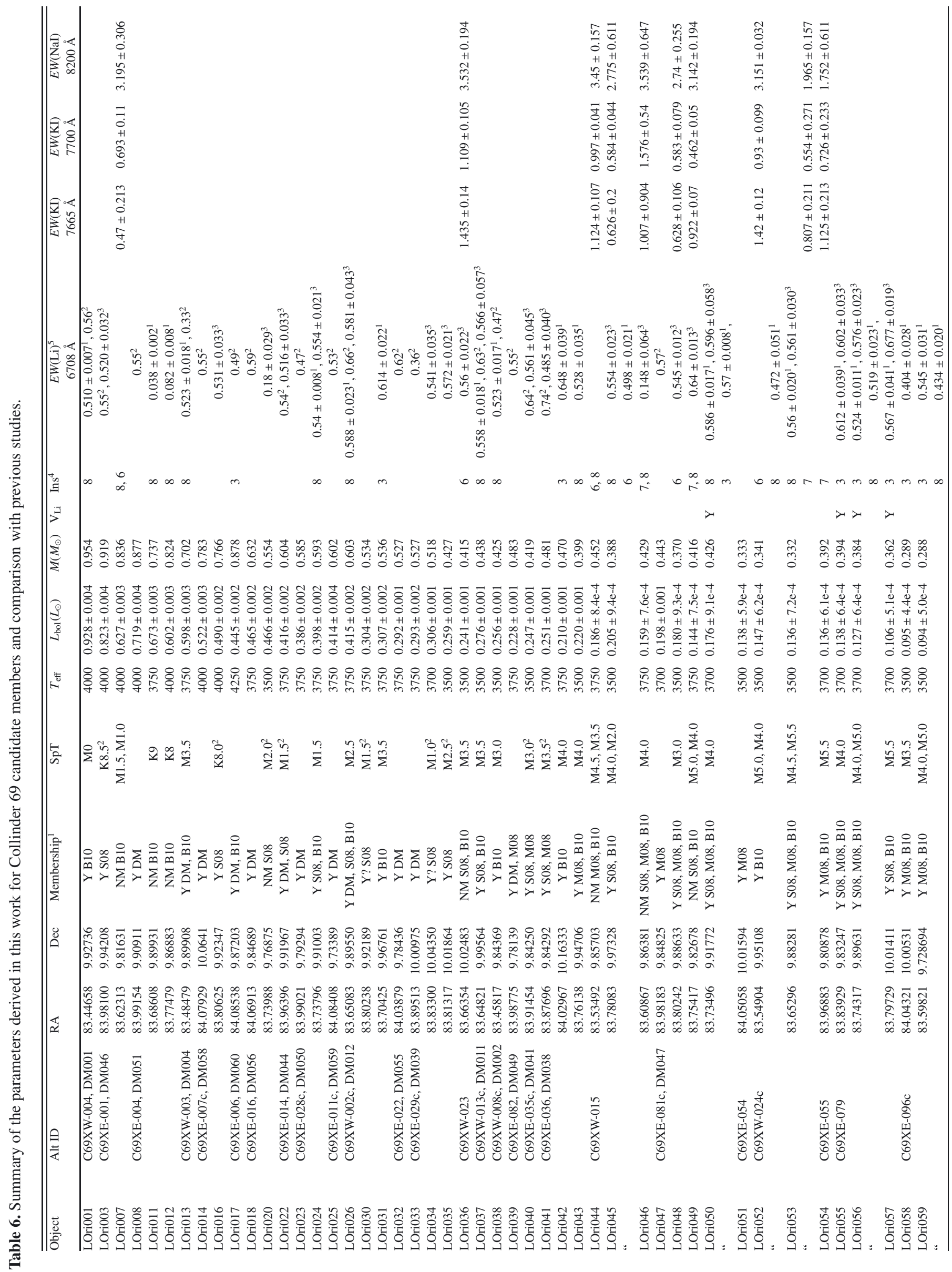




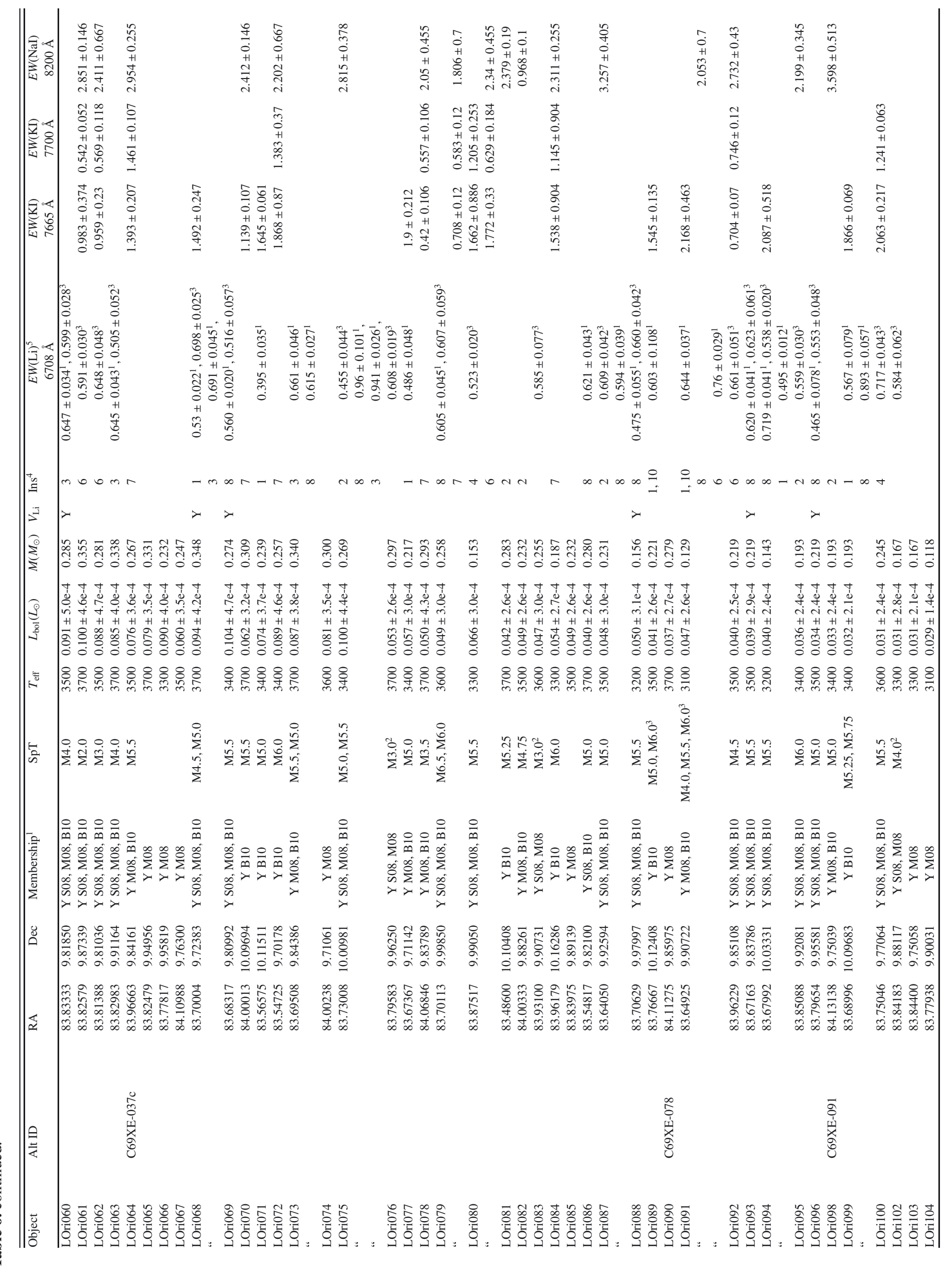


A. Bayo et al.: Spectroscopy of very low mass stars and brown dwarfs in the Lambda Orionis star forming region

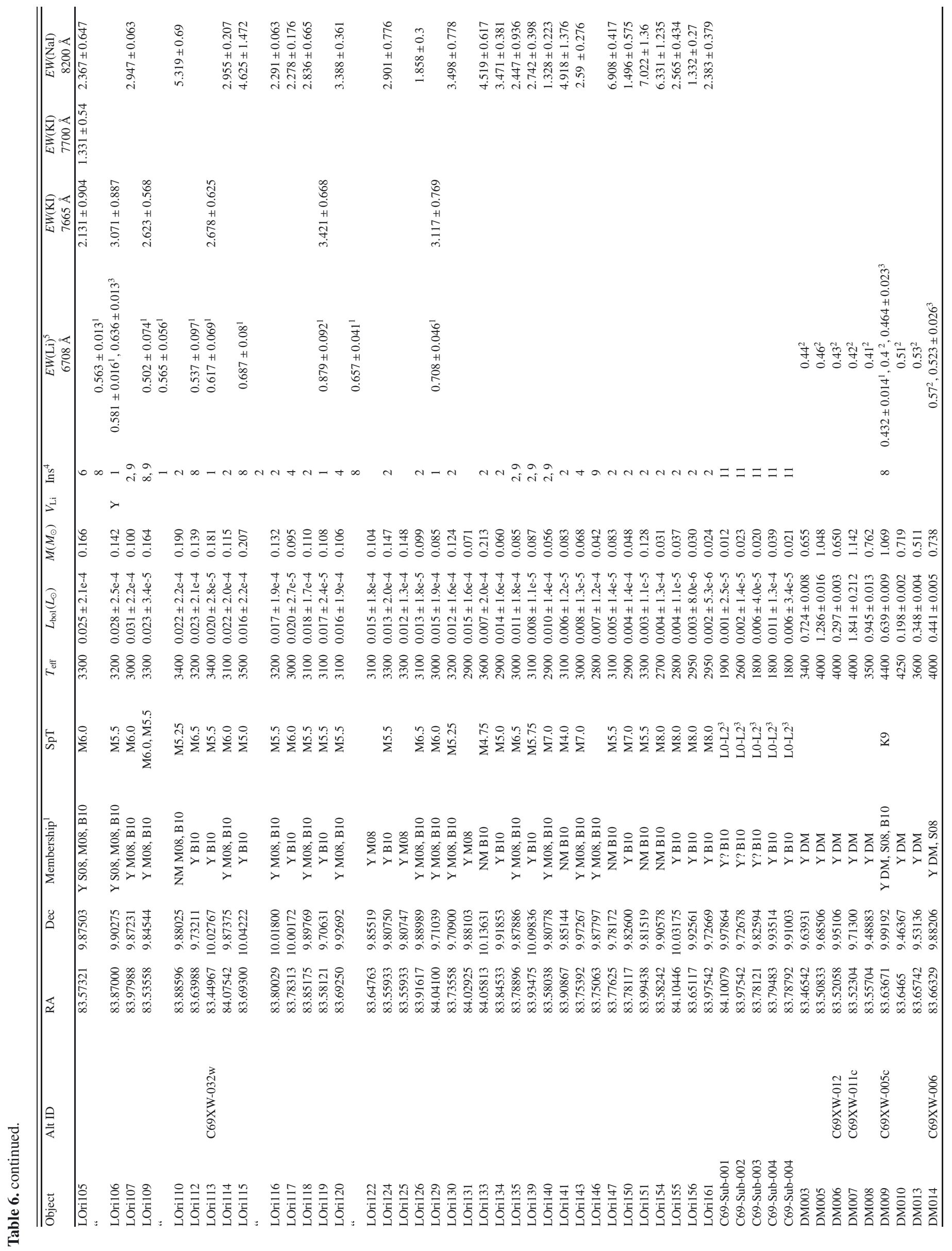


A\&A 536, A63 (2011)

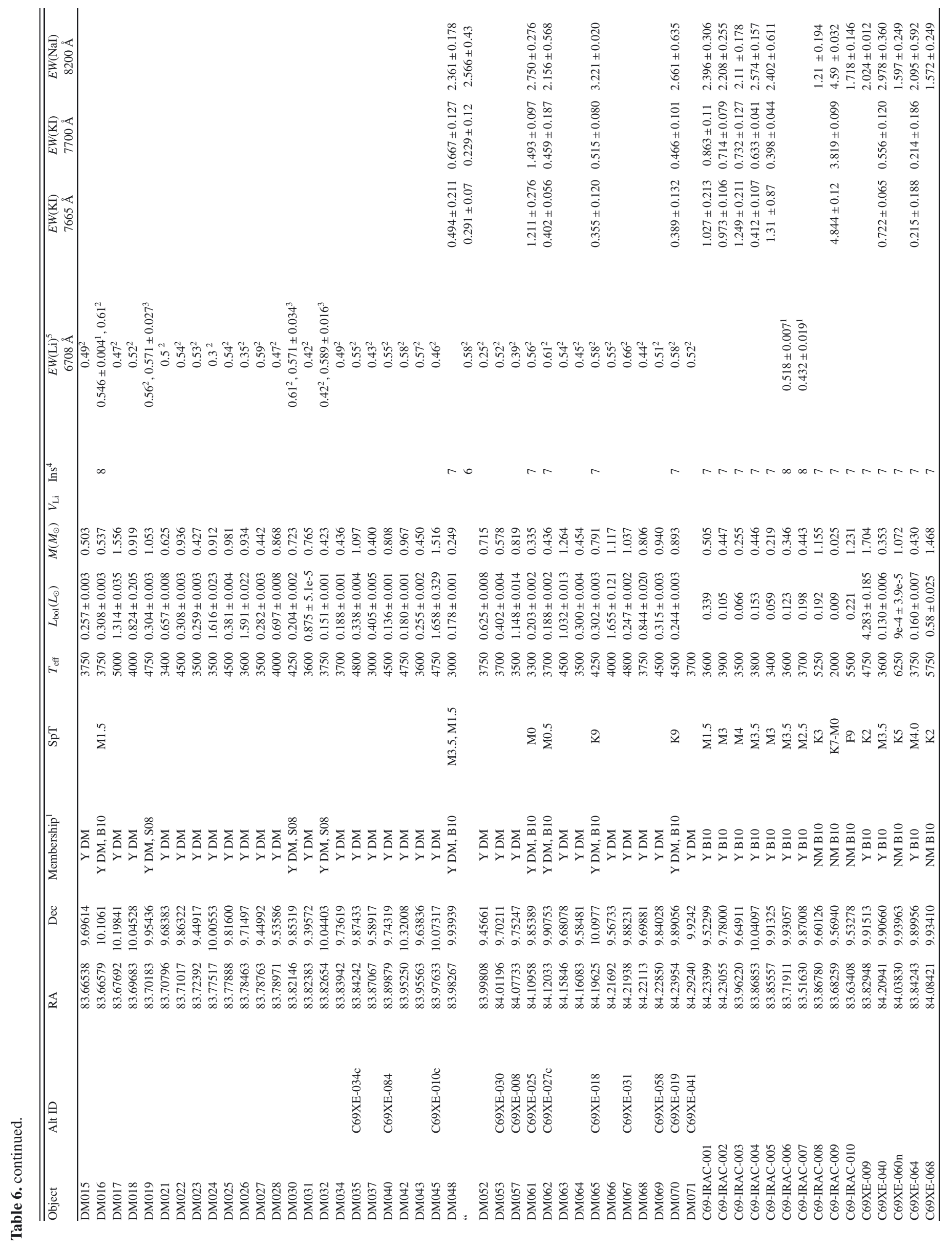

A63, page 22 of 23 
A. Bayo et al.: Spectroscopy of very low mass stars and brown dwarfs in the Lambda Orionis star forming region

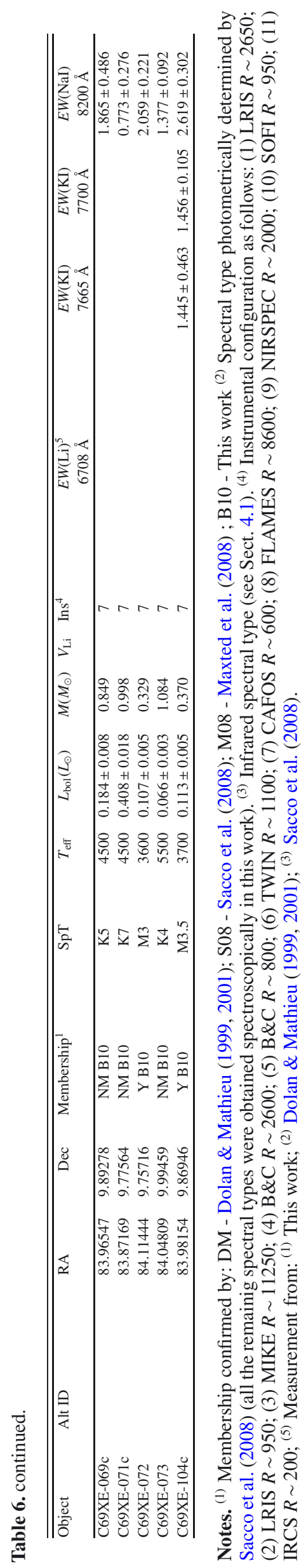

